Pacific

Journal of

Mathematics

\title{
INVARIANT DIFFERENTIAL OPERATORS ON A CLASS OF MULTIPLICITY-FREE SPACES
}

HuberT RubENTHALER 


\title{
INVARIANT DIFFERENTIAL OPERATORS ON A CLASS OF MULTIPLICITY-FREE SPACES
}

\author{
HUBERT RUBENTHALER
}

\begin{abstract}
If $(G, V)$ is a multiplicity-free space with a one-dimensional quotient, we give generators and relations for the noncommutative algebra $D(V)^{G^{\prime}}$ of invariant differential operators under the semisimple part $G^{\prime}$ of the reductive group $G$. More precisely we show that $D(V)^{G^{\prime}}$ is the quotient of a Smith algebra by a completely described two-sided ideal.
\end{abstract}

\section{Introduction}

Let $H$ be a reductive algebraic group over $\mathbb{C}$ and let $X$ be a smooth irreducible $H$-variety. Let $\mathbb{C}[X]$ be the algebra of regular functions on $X$ and let $D(X)$ be the algebra of differential operators on $X$. Then the $H$-action on $X$ extends naturally to $\mathbb{C}[X]$ and $D(X)$. Let $\mathbb{C}[X]^{H}$ (resp. $D(X)^{H}$ ) be the subalgebras of $H$-invariants in $\mathbb{C}[X]$ (resp. $D(X))$. The ring $\mathbb{C}[X]^{H}$ is the ring of regular functions on the categorical quotient $X / / H$. The problem of determining the structure of $D(X)^{H}$ was investigated by several authors [Levasseur and Stafford 1989; Van den Bergh 1996; Schwarz 2002]. On the other hand under the above mentioned hypothesis there exists an $H$-equivariant restriction map

$$
\delta: D(X)^{H} \rightarrow D(X / / H),
$$

obtained by applying elements in $D(X)^{H}$ to functions in $\mathbb{C}[X]^{H}$. It is expected that $D(X)^{H}$, as well as its image under $\delta$ (the so-called algebra of radial components), should share many properties of enveloping algebras [Schwarz 2002; Levasseur 2009]. In this paper we obtain the precise structure of $D(V)^{G^{\prime}}$ in the case where $(G, V)$ is a so-called multiplicity-free space with a one-dimensional quotient (here $G$ is reductive and $G^{\prime}=[G, G]$ is the derived group). These spaces are defined to be the multiplicity-free spaces $(G, V)$ for which the quotient $V / / G^{\prime}$ is one-dimensional. To be more precise we show that the (noncommutative) algebra $D(V)^{G^{\prime}}$ is a quotient of a generalized Smith algebra. Over $\mathbb{C}$ this kind of algebra was introduced by S. P. Smith [1990] as a natural generalization of the enveloping algebra of $\mathfrak{s l}_{2}$. As a corollary we describe by generators and relations the algebras of radial components

MSC2010: primary 22E46, 16S32; secondary 11S90.

Keywords: multiplicity-free space, invariant differential operator, Smith algebra. 
attached to the $G^{\prime}$-isotypic components in the polynomial algebra $\mathbb{C}[V]$ (the image under $\delta$ above corresponds to the trivial representation of $G^{\prime}$ ).

According to the classification obtained in [Rubenthaler 2013], the class of multiplicity-free spaces with a one-dimensional quotient is a rather large class inside the multiplicity-free spaces. It contains both irreducible and nonirreducible representations.

The representations $(\operatorname{Str}(V), V)$, where $V$ is a simple Jordan algebra over $\mathbb{C}$ and where $\operatorname{Str}(V)$ is the structure group of $V$, are examples of irreducible multiplicityfree spaces with a one-dimensional quotient (see Remark 2.2.7 and Example 2.3.3 below). Among these there is the natural representation of $\operatorname{GL}(n, \mathbb{C})$ on the space $\operatorname{Sym}_{n}(\mathbb{C})$ of $n \times n$ symmetric matrices and also the irreducible 27-dimensional representation of $E_{6} \times \mathbb{C}^{*}$.

The spin representation of $\operatorname{Spin}(7) \times \mathbb{C}^{*}$ and the irreducible 7-dimensional representation of $G_{2} \times \mathbb{C}^{*}$ are other irreducible examples.

The representation $\left(\operatorname{SL}(n, \mathbb{C}) \times\left(\mathbb{C}^{*}\right)^{2}, \Lambda_{1} \oplus \Lambda^{2}\left(\Lambda_{1}\right)\right)(n$ odd and $n \geq 5)$, where $\Lambda_{1}$ is the natural representation of $\operatorname{SL}(n, \mathbb{C})$ and $\Lambda^{2}\left(\Lambda_{1}\right)$ is its second exterior power, provides a nonirreducible example.

Let us now give a more precise description of our paper.

In Section 2 we give basic definitions and properties of, and notation for, multiplicity-free spaces, including multiplicity-free spaces with a one-dimensional quotient. If $(G, V)$ is a multiplicity-free space then $G$ has an open orbit on $V$ (i.e., $(G, V)$ is a prehomogeneous vector space). We also prove that in the so-called regular case the $G$-invariant differential operators on the open orbit of a multiplicityfree space always have polynomial coefficients (in fact a slightly more general result is proved; see Theorem 2.2.6).

In Section 3 we introduce the various algebras of differential operators we are interested in. We define their natural gradings and we define the so-called BernsteinSato polynomial of a homogeneous operator of any degree, not only for degree-zero operators as usual. We obtain there the first results concerning these algebras. Using the Harish-Chandra isomorphism for multiplicity-free spaces [Knop 1998], we prove a key lemma on invariant polynomials under the so-called little Weyl group which enables us to prove that $D(V)^{G}$ is a polynomial algebra over the center $\mathscr{L}(\mathscr{T})$ of $D(V)^{G^{\prime}}$, with the Euler operator as generator (Theorem 3.2.6). We also give generators of the center $\mathscr{E}(\mathscr{T})$ (Theorem 3.2.10) and obtain some specific results in the case of prehomogeneous vector spaces of commutative parabolic type (Theorem 3.3.1).

Section 4, which is the main section, is devoted to the structure of $D(V)^{G^{\prime}}$. We first briefly define and study Smith algebras over a commutative ring $\boldsymbol{A}$ with unit and no zero divisors (the original definition by Smith was over $\mathbb{C}$ ). These algebras are defined by generators and relations (involving a polynomial in $\boldsymbol{A}[t]$ ), and their 
center is a polynomial algebra $A\left[\Omega_{1}\right]$, where $\Omega_{1}$ is a generalized Casimir element. Our main result asserts that $D(V)^{G^{\prime}}$ is isomorphic to the quotient of a Smith algebra over its center $\mathscr{L}(\mathscr{T})$ by the two-sided ideal generated by the element $\Omega_{1}$. Concretely, we give generators and relations for $D(V)^{G^{\prime}}$ (see Theorem 4.2.2).

Section 5 is devoted to the study of the algebras of radial components. By radial component of a differential operator in $D(V)^{G^{\prime}}$ we mean the restriction of $D$ to a $G^{\prime}$-isotypic component of $\mathbb{C}[V]$. As a corollary of the preceding results we prove that these algebras are quotients of "classical" Smith algebras, that is, Smith algebras over $\mathbb{C}$ (see Theorem 5.2.3). Of course the defining relations depend on the $G^{\prime}$-isotypic component. We also give generators of the kernel of the radial component map. In the case of the trivial representation of $G^{\prime}$, the structure of the algebra of radial components was first obtained by Levasseur [2009], by other methods.

\section{Multiplicity-free spaces with a one-dimensional quotient}

2.1. Prehomogeneous vector spaces, basic definitions and properties. Let $G$ be a connected algebraic group over $\mathbb{C}$, and let $(G, \rho, V)$ be a rational representation of $G$ on the (finite-dimensional) vector space $V$. Then the triplet $(G, \rho, V)$ is called a prehomogeneous vector space (abbreviated to PV) if the action of $G$ on $V$ has a Zariski open orbit $\Omega \subset V$. For the general theory of PVs, we refer the reader to the book of Kimura [2003] or to [Sato and Kimura 1977]. The elements in $\Omega$ are called generic. The PV is said to be irreducible if the corresponding representation is irreducible. The singular set $S$ of $(G, \rho, V)$ is defined by $S=V \backslash \Omega$. Elements in $S$ are called singular. If no confusion can arise we often simply denote the PV by $(G, V)$. We will also write $g . x$ instead of $\rho(g) x$, for $g \in G$ and $x \in V$. It is easy to see that the condition for a rational representation $(G, \rho, V)$ to be a PV is in fact an infinitesimal condition. More precisely let $\mathfrak{g}$ be the Lie algebra of $G$ and let $d \rho$ be the derived representation of $\rho$. Then $(G, \rho, V)$ is a PV if and only if there exists $v \in V$ such that the map

$$
\begin{aligned}
\mathfrak{g} & \rightarrow V, \\
X & \mapsto d \rho(X) v,
\end{aligned}
$$

is surjective (we will often write $X . v$ instead of $d \rho(X) v)$. Therefore we will call $(\mathfrak{g}, V)$ a PV if the preceding condition is satisfied.

Let $(G, V)$ be a PV. A rational function $f$ on $V$ is called a relative invariant of $(G, V)$ if there exists a rational character $\chi$ of $G$ such that $f(g . x)=\chi(g) P(x)$ for $g \in G$ and $x \in V$. From the existence of an open orbit it is easy to see that a character $\chi$ which is trivial on the isotropy subgroup of an element $x \in \Omega$ determines a unique relative invariant $P$. Let $S_{1}, S_{2}, \ldots, S_{k}$ denote the irreducible components of codimension one of the singular set $S$. Then there exist irreducible polynomials 
$P_{1}, P_{2}, \ldots, P_{k}$ such that $S_{i}=\left\{x \in V \mid P_{i}(x)=0\right\}$. The polynomials $P_{i}$ are unique up to nonzero constants; they are relative invariants of $(G, V)$ and any nonzero relative invariant $f$ can be written in a unique way as $f=c P_{1}^{n_{1}} P_{2}^{n_{2}} \cdots P_{k}^{n_{k}}$, where $n_{i} \in \mathbb{Z}$ and $c \in \mathbb{C}^{*}$. The polynomials $P_{1}, P_{2}, \ldots, P_{k}$ are called the fundamental relative invariants of $(G, V)$. Moreover if the representation $(G, V)$ is irreducible then there exists at most one irreducible polynomial which is relatively invariant.

The prehomogeneous vector space $(G, V)$ is called regular if there exists a relative invariant polynomial $P$ whose Hessian $H_{P}(x)$ is nonzero on $\Omega$. If $G$ is reductive, then $(G, V)$ is regular if and only if the singular set $S$ is a hypersurface, or if and only if the isotropy subgroup of a generic point is reductive. If the $\mathrm{PV}(G, V)$ is regular, or if $G$ is reductive, then the contragredient representation $\left(G, V^{*}\right)$ is again a PV.

2.2. Multiplicity-free spaces. For the results concerning multiplicity-free spaces we refer the reader to the survey by Benson and Ratcliff [2004] or to [Knop 1998]. Let $(G, V)$ be a finite-dimensional rational representation of a connected reductive algebraic group $G$. Let $\mathbb{C}[V]$ be the algebra of polynomials on $V$. Then $G$ acts on $\mathbb{C}[V]$ by

$$
g . \varphi(x)=\varphi\left(g^{-1} x\right) \quad(g \in G, \varphi \in \mathbb{C}[V]) .
$$

As the space $\mathbb{C}[V]^{n}$ of homogeneous polynomials of degree $n$ is stable under this action, the representation $(G, \mathbb{C}[V])$ is completely reducible. Let $D(V)$ be the algebra of differential operators on $V$ with polynomial coefficients. The group $G$ acts also on $D(V)$ by

$$
(g . D)(\varphi)=g .\left(D\left(g^{-1} . \varphi\right)\right) \quad(g \in G, D \in D(V), \varphi \in \mathbb{C}[V]) .
$$

Recall the $G$-equivariant identifications between $\mathbb{C}[V]$ and the symmetric algebra $S\left(V^{*}\right)$ of the dual space $V^{*}$ and between $\mathbb{C}\left[V^{*}\right]$ and the symmetric algebra $S(V)$ of $V$. The embedding

$$
\begin{aligned}
& V \rightarrow D(V), \\
& v \mapsto D_{v},
\end{aligned}
$$

where $D_{v} P(x)=\lim _{t \rightarrow 0}(P(x+t v)-P(x)) / t$, extends uniquely to an embedding $S(V) \rightarrow D(V)$ whose image is the ring of differential operators with constant coefficients. If $f \in S(V) \simeq \mathbb{C}\left[V^{*}\right]$ we denote by $f(\partial)$ the corresponding differential operator. Another way to construct $f(\partial)$ for $f \in \mathbb{C}\left[V^{*}\right]$ is to say that $f(\partial)$ is the unique differential operator on $V$ satisfying

$$
f\left(\partial_{x}\right) e^{\langle x, y\rangle}=f(y) e^{\langle x, y\rangle} \quad\left(x \in V, y \in V^{*}\right) .
$$


Recall also that the $\mathbb{C}[V]$-module $D(V)$ can be identified with $\mathbb{C}[V] \otimes S(V)$ through the multiplication map

$$
\begin{aligned}
m: \mathbb{C}[V] \otimes S(V) & \stackrel{\simeq}{\longrightarrow} D(V), \\
\varphi \otimes f & \longmapsto \varphi f(\partial) .
\end{aligned}
$$

The preceding map is in fact $G$-equivariant and therefore the $G$-module $D(V)$ is isomorphic to the $G$-module $\mathbb{C}[V] \otimes S(V)$. The duality pairing $V \otimes V^{*} \rightarrow \mathbb{C}$ extends uniquely to the nondegenerate $G$-equivariant pairing

$$
\begin{aligned}
S(V) \otimes S\left(V^{*}\right) \simeq \mathbb{C}\left[V^{*}\right] \otimes \mathbb{C}[V] & \rightarrow \mathbb{C}, \\
f \otimes \varphi & \mapsto\langle f, \varphi\rangle=f(\partial) \varphi(0),
\end{aligned}
$$

which gives rise to an embedding $\mathbb{C}\left[V^{*}\right] \hookrightarrow \mathbb{C}[V]^{*}$. It is easy to see that if $i \neq j$, $\left\langle\mathbb{C}^{i}\left[V^{*}\right], \mathbb{C}^{j}[V]\right\rangle=\{0\}$.

Definition 2.2.1. Let $G$ be a connected reductive algebraic group, and let $V$ be the space of a finite-dimensional (complex) rational representation of $G$. The representation $(G, V)$ is said to be multiplicity-free (abbreviated to $M F$ ) if each irreducible representation of $G$ occurs at most once in the representation $(G, \mathbb{C}[V])$.

Remark 2.2.2. Historically the classification of MF spaces goes as follows. Kac [1980] determined all the MF spaces where the representation $(G, V)$ is irreducible. Brion [1985] did the case where $G^{\prime}=[G, G]$ is (almost) simple. Finally, Benson and Ratcliff [1996; 2004] and independently Leahy [1998] (see also [Knop 1998]) classified all indecomposable saturated MF spaces up to geometric equivalence.

The following theorem summarizes some basic results concerning MF spaces (see [Howe and Umeda 1991; Knop 1998; Benson and Ratcliff 2004]):

Theorem 2.2.3. 1) A finite-dimensional representation $(G, V)$ is $M F$ if and only if $(B, V)$ is a prehomogeneous vector space for any Borel subgroup $B$ of $G$ (and hence each $M F$ space $(G, V)$ is a $P V)$.

2) A finite-dimensional representation $(G, V)$ is $M F$ if and only if the algebra $D(V)^{G}$ of invariant differential operators with polynomial coefficients is commutative.

3) If $(G, V)$ is a MF space, then the dual space $\left(G, V^{*}\right)$ is also $M F$.

Proof. The first assertion is due to [Vinberg and Kimelfeld 1978]; another proof can be found in [Knop 1998]. The second assertion is due to [Howe and Umeda 1991, Theorem 7.1]. For the third assertion note that as $\left\langle\mathbb{C}^{i}\left[V^{*}\right], \mathbb{C}^{j}[V]\right\rangle=\{0\}$ for $i \neq j$, we obtain that $f \mapsto\langle f, \quad\rangle$ is a $G$-equivariant isomorphism between $\mathbb{C}^{i}\left[V^{*}\right]$ and $\mathbb{C}^{i}[V]^{*}$, and hence $\left(G, V^{*}\right)$ is multiplicity-free. 
Let us be more precise about the decomposition of the polynomials under the action of the group $G$ or a Borel subgroup. Therefore we need more notation. We can write $G=G^{\prime} C$, where $G^{\prime}=[G, G]$ is the subgroup of commutators and $C=Z(G)^{\circ} \simeq\left(\mathbb{C}^{*}\right)^{p}$ is the connected component of the center of $G$. Let $T^{\prime}$ be a maximal torus in $G^{\prime}$, and let $B^{\prime}=T^{\prime} U$ be a Borel subgroup of $G^{\prime}$, where $U$ is the nilradical of $B^{\prime}$. The group $T=T^{\prime} C$ is a maximal torus in $G$ and $B=T U$ is a Borel subgroup of $G$. We will denote by $\mathfrak{g}, \mathfrak{g}^{\prime}, \mathfrak{t}, \mathfrak{t}^{\prime}, \mathfrak{c}, \mathfrak{b}, \mathfrak{b}^{\prime}, \mathfrak{u}$ the corresponding Lie algebras. Let $R$ be the set of roots of $\left(\mathfrak{g}^{\prime}, \mathfrak{t}^{\prime}\right)$, let $\Delta=\left\{\alpha_{1}, \ldots, \alpha_{\ell}\right\}$ be the basis of simple roots corresponding to $\mathfrak{b}^{\prime}$ and let $R^{+}$be the corresponding set of positive roots.

Denote by $\Lambda^{\prime}$ the lattice of weights of $\left(\mathfrak{g}^{\prime}, \mathfrak{t}^{\prime}\right)$. Then $\Lambda^{\prime}=\mathbb{Z} \omega_{1} \oplus \mathbb{Z} \omega_{2} \oplus \cdots \oplus \mathbb{Z} \omega_{\ell}$, where the $\omega_{i}$ are the fundamental weights. Let $\Lambda^{\prime+}=\mathbb{N} \omega_{1} \oplus \mathbb{N} \omega_{2} \oplus \cdots \oplus \mathbb{N} \omega_{\ell}$ be the set of dominant weights. Denote by $X(C)$ the group of algebraic characters of $C$, which we will sometimes consider as linear forms on $\mathfrak{c}$. Set

$$
\Lambda=\Lambda^{\prime} \oplus X(C), \quad \Lambda^{+}=\Lambda^{\prime+} \oplus X(C) .
$$

For $\lambda \in \Lambda^{+}$(resp. $\lambda^{\prime} \in \Lambda^{\prime+}$ ) let us denote by $V_{-\lambda}$ (resp. $V_{-\lambda^{\prime}}$ ) an irreducible $\mathfrak{g}$-module (resp. $\mathfrak{g}^{\prime}$-module) with the highest weight $\lambda$ (resp. $\lambda^{\prime}$ ). We use this unusual notation because we want to index the modules occurring in $\mathbb{C}[V]$ by the character of their highest weight polynomial, rather than by the highest weight.

For a multiplicity-free space $(G, V)$ we have the decomposition

$$
\mathbb{C}[V]=\bigoplus_{\lambda \in \Lambda^{+}} V_{-\lambda}^{m(\lambda)}
$$

where $m(\lambda)=0$ or 1 . If $m(\lambda)=1$, then there exists a uniquely defined positive integer $d(\lambda)$ such that $V_{-\lambda} \in \mathbb{C}[V]^{d(\lambda)}$. The integer $d(\lambda)$ is called the degree of $\lambda$. Let us denote by $\Delta_{0}, \Delta_{1}, \ldots, \Delta_{k}, \ldots, \Delta_{r}$ the fundamental relative invariants of the PV $(B, V)$, indexed in such a way that $\Delta_{0}, \Delta_{1}, \ldots, \Delta_{k}$ are the fundamental relative invariants of the $\mathrm{PV}(G, V)$ and such that the other invariants are ordered by decreasing degree. We denote by $d_{i}$ the degree of $\Delta_{i}(i=0, \ldots, r)$. It is worthwhile noticing that at least $\Delta_{r}$ is of degree one as the highest weight vectors of the irreducible components of $V^{*}$ must occur. Then any relative invariant of $(B, V)$ is of the form $c \Delta^{\boldsymbol{a}}$, where $\boldsymbol{a}=\left(a_{0}, a_{1}, \ldots, a_{r}\right) \in \mathbb{Z}^{r+1}$ and $\Delta^{\boldsymbol{a}}=\Delta_{0}^{a_{0}} \cdots \Delta_{r}^{a_{r}}$. The nonnegative integer $r+1$ is called the rank of the MF space $(G, V)$. The algebra of $U$-invariants is the subalgebra generated by the $\Delta_{i}$; i.e., $\mathbb{C}[V]^{U}$ is given by $\mathbb{C}\left[\Delta_{0}, \ldots, \Delta_{r}\right]$. As the polynomials $\Delta_{i}$ are algebraically independent, this latter algebra is a polynomial algebra. Let $\lambda_{i}$ be the character of $\Delta_{i}$ (we use the same notation $\lambda_{i}$ for the character of the group and for its derivative, which is an element of $\Lambda^{+}$). Hence the (infinitesimal) character of $\Delta^{a}$ is $\lambda_{\boldsymbol{a}}=a_{0} \lambda_{0}+\cdots+a_{r} \lambda_{r}$. Of course by definition the elements $\Delta^{a}\left(a_{i} \geq 0, i=0, \ldots, r\right)$ are the highest weights 
vectors in $\mathbb{C}[V]$. Due to the fact that the group action on $\Delta^{a}$ is given by $g . \Delta^{a}(x)=$ $\Delta^{a}\left(g^{-1} x\right)$, the infinitesimal highest weight of $\Delta^{a}$ is $-\lambda_{a}=-a_{0} \lambda_{0}-\cdots-a_{r} \lambda_{r}$.

If we set $V_{\boldsymbol{a}}=V_{-\lambda_{a}}$, we therefore can write

$$
\mathbb{C}[V]=\bigoplus_{a_{0} \geq 0, \ldots, a_{r} \geq 0} V_{\boldsymbol{a}}
$$

Sometimes, if $\lambda=a_{0} \lambda_{0}+\cdots+a_{r} \lambda_{r}$, we simply write $V_{\lambda}$ instead of $V_{\boldsymbol{a}}$. If we denote by $d_{i}$ the degree of $\Delta_{i}$, one can notice that all elements in $V_{\boldsymbol{a}}$ are of degree $d(\boldsymbol{a})=a_{0} d_{0}+a_{1} d_{1}+\cdots+a_{r} d_{r}$. It is also worthwhile noticing that we have

$$
V_{\boldsymbol{a}}=\Delta_{0}^{a_{0}} \Delta_{1}^{a_{1}} \cdots \Delta_{k}^{a_{k}} V_{0, \ldots, 0, a_{k+1}, \ldots, a_{r}} .
$$

The proof of the following lemma is straightforward.

Lemma 2.2.4. Define $\mathcal{O}=\left\{x \in V \mid \Delta_{i}(x) \neq 0, i=0, \ldots, k\right\}$. Let $\mathbb{C}[\mathcal{O}]$ be the ring of regular functions on $\mathbb{O}$ (elements of $\mathbb{C}[0]$ are just rational functions whose denominators are of the form $\Delta_{0}^{a_{0}} \cdots \Delta_{k}^{a_{k}}$, with $\left.a_{0}, \ldots, a_{k} \geq 0\right)$. As the polynomials $\Delta_{0}, \ldots, \Delta_{k}$ are relative invariants under $G$, the open set $\mathcal{O}$ is $G$-stable, and therefore $G$ acts on $\mathbb{C}[\mathbb{O}]$. Then $\mathbb{C}[\mathbb{O}]$ decomposes without multiplicities under the action of $G$. More precisely the decomposition into irreducibles is given by

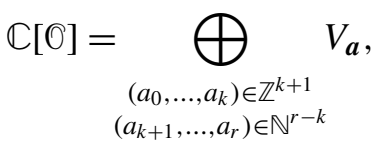

where $V_{\boldsymbol{a}}=\Delta_{0}^{a_{0}} \Delta_{1}^{a_{1}} \cdots \Delta_{k}^{a_{k}} V_{0, \ldots, 0, a_{k+1}, \ldots, a_{r}}$ is the irreducible subspace of $\mathbb{C}[\mathbb{O}]$ generated by the highest weight vector $\Delta^{a}=\Delta_{0}^{a_{0}} \Delta_{1}^{a_{1}} \cdots \Delta_{r}^{a_{r}}$.

Remark 2.2.5. We want to draw the attention of the reader to the fact that if $(G, V)$ is not a regular PV, then the open set 0 may be distinct from the open $G$-orbit $\Omega$.

The preceding lemma has the following consequence.

Theorem 2.2.6. Let $(G, V)$ be a multiplicity-free space. As before set

$$
\mathcal{O}=\left\{x \in V \mid \Delta_{i}(x) \neq 0, i=0, \ldots, k\right\} .
$$

Then $D(V)^{G}=D(O)^{G}$. In other words any $G$-invariant differential operator with coefficients in $\mathbb{C}[\mathbb{O}]$ has in fact polynomial coefficients.

Proof. Let $D \in D(\mathcal{O})^{G}$. As we know from the preceding lemma that $\mathbb{C}[\mathcal{O}]$ decomposes without multiplicities under $G$, we obtain that $D$ defines a $G$-equivariant endomorphism on each $V_{\boldsymbol{a}}, \boldsymbol{a} \in \mathbb{Z}^{k+1} \times \mathbb{N}^{r-k}$. Thus $D$ stabilizes $\mathbb{C}[V]=\bigoplus_{a_{0} \geq 0, \ldots, a_{r} \geq 0} V_{\boldsymbol{a}}$. It is easy to see that a differential operator with rational coefficients and which stabilizes the polynomials must have polynomial coefficients. 
Remark 2.2.7. Let $V$ be a simple Jordan algebra over $\mathbb{C}$ or $\mathbb{R}$. Let $\Omega$ be the set of invertible elements in $V$ and let $G$ be the structure group of $V$. It is known that $(G, V)$ is a multiplicity-free space with $\Omega$ as open $G$-orbit. Then the preceding theorem implies that $D(V)^{G}=D(\Omega)^{G}$. This result was already known in this context and is usually obtained by computing an explicit set of generators of $D(\Omega)^{G}$ (see [Nomura 1989; Faraut and Korányi 1994; Yan 2000]). Through the so-called Kantor-Koecher-Tits construction there is a one-to-one correspondence between these spaces and the PVs of commutative parabolic type (see Example 2.3.3 below).

Proposition 2.2.8. Let $(G, V)$ be a MF space. For $\tilde{\boldsymbol{a}}=\left(a_{k+1}, \ldots, a_{r}\right) \in \mathbb{N}^{r-k}$ we define $V_{\tilde{\boldsymbol{a}}}=V_{\left(0, \ldots, 0, a_{k+1}, \ldots, a_{r}\right)}$. Then for $\boldsymbol{a}=\left(a_{0}, \ldots, a_{k}, a_{k+1}, \ldots, a_{r}\right)$ the spaces $V_{\boldsymbol{a}}=\Delta_{0}^{a_{0}} \cdots \Delta_{k}^{a_{k}} V_{\tilde{\boldsymbol{a}}}$ are $G^{\prime}$-equivalent if $\tilde{\boldsymbol{a}}$ is fixed and if $\left(a_{0}, \ldots, a_{k}\right) \in \mathbb{Z}^{k+1}$. If we define

$$
U_{\tilde{\boldsymbol{a}}}=\bigoplus_{\left(a_{0}, \ldots, a_{k}\right) \in \mathbb{N}^{k+1}} \Delta_{0}^{a_{0}} \cdots \Delta_{k}^{a_{k}} V_{\tilde{\boldsymbol{a}}}, \quad W_{\tilde{\boldsymbol{a}}}=\bigoplus_{\left(a_{0}, \ldots, a_{k}\right) \in \mathbb{Z}^{k+1}} \Delta_{0}^{a_{0}} \cdots \Delta_{k}^{a_{k}} V_{\tilde{\boldsymbol{a}}},
$$

the decompositions of $\mathbb{C}[V]$ and $\mathbb{C}[0]$ into $G^{\prime}$-isotypic components are given by

$$
\mathbb{C}[V]=\bigoplus_{\tilde{\boldsymbol{a}}} U_{\tilde{\boldsymbol{a}}}, \quad \mathbb{C}[\mathrm{O}]=\bigoplus_{\tilde{\boldsymbol{a}}} W_{\tilde{\boldsymbol{a}}}
$$

Proof. The map $P \mapsto \Delta_{0}^{a_{0}} \cdots \Delta_{k}^{a_{k}} P$ is a $G^{\prime}$-equivariant isomorphism between $V_{\tilde{\boldsymbol{a}}}$ and $\Delta_{0}^{a_{0}} \cdots \Delta_{k}^{a_{k}} V_{\tilde{\boldsymbol{a}}}$; hence all these spaces are $G^{\prime}$-equivalent. To prove the second assertion it is enough to prove that if $\tilde{\boldsymbol{a}} \neq \tilde{\boldsymbol{b}}$, then the spaces $V_{\tilde{\boldsymbol{a}}}$ and $V_{\tilde{\boldsymbol{b}}}$ are not $G^{\prime}$-equivalent. Suppose that this would be the case and let $\Delta^{\tilde{a}}$ and $\Delta^{\tilde{b}}$ be the corresponding highest weight vectors with characters $\lambda_{\tilde{a}}$ and $\lambda_{\tilde{b}}$ respectively. From the $G^{\prime}$-equivalence we know that $\left.\lambda_{\tilde{\boldsymbol{a}}}\right|_{\mathfrak{t}^{\prime}}=\left.\lambda_{\tilde{\boldsymbol{b}}}\right|_{\mathfrak{t}^{\prime}}$ and hence $P=\Delta^{\tilde{\boldsymbol{a}}} / \Delta^{\tilde{\boldsymbol{b}}}$ is a relative invariant under $B$ whose character is trivial on $\mathfrak{t}^{\prime}$. Therefore it generates a onedimensional representation; hence $P$ is a relative invariant under $G$. Finally we obtain that $\Delta^{\tilde{\boldsymbol{a}}}=\Delta_{0}^{a_{0}} \cdots \Delta_{k}^{a_{k}} \Delta^{\tilde{\boldsymbol{b}}}$, and this is not possible if $\tilde{\boldsymbol{a}} \neq \tilde{\boldsymbol{b}}$.

As $\left(G, V^{*}\right)$ is multiplicity-free (Theorem 2.2.3) and $\mathbb{C}^{i}\left[V^{*}\right] \simeq \mathbb{C}^{i}[V]^{*}$, we have

$$
\mathbb{C}\left[V^{*}\right]=\bigoplus_{a_{0} \geq 0, \ldots, a_{r} \geq 0} V_{\boldsymbol{a}}^{*}
$$

where $V_{\boldsymbol{a}}^{*}$ is the irreducible $G$-submodule of $\mathbb{C}\left[V^{*}\right]$ generated by a lowest weight vector $\Delta^{* a} \in \mathbb{C}\left[V^{*}\right]$, defined up to a multiplicative constant, whose character with respect to the opposite Borel subgroup $B^{-}$is equal to $-\lambda_{a}=-a_{0} \lambda_{0}-\cdots-a_{r} \lambda_{r}$. Let us fix a lowest weight vector $\Delta_{i}^{*}(i=0, \ldots, r)$ with character $-\lambda_{i}$ (with respect to $B^{-}$). Then we can choose $\Delta^{* \boldsymbol{a}}=\Delta_{0}^{* a_{0}} \Delta_{1}^{* a_{1}} \cdots \Delta_{r}^{* a_{r}}$. Of course the module $V_{\boldsymbol{a}}^{*}$ is the dual module of $V_{\boldsymbol{a}}$ through $f \mapsto\langle f, \quad\rangle$ (see (2-2-2)). 
As $V_{\boldsymbol{a}}$ is a $G$-irreducible module, it is well known that the tensor $G$-module $V_{\boldsymbol{a}} \otimes V_{\boldsymbol{a}}^{*}$ contains, up to a constant, a unique $G$-invariant vector $R_{\boldsymbol{a}}$ and that $V_{\boldsymbol{a}} \otimes V_{\boldsymbol{b}}^{*}$ does not contain any nontrivial $G$-invariant vector if $\boldsymbol{a} \neq \boldsymbol{b}$ (see for example [Howe and Umeda 1991]). To be more precise we define $R_{a}$ to be the operator corresponding to the "unit matrix" in $V_{a} \otimes V_{a}^{*} \simeq \operatorname{Hom}\left(V_{a}, V_{a}\right)$. Moreover as $\mathbb{C}[V] \otimes \mathbb{C}\left[V^{*}\right]$ is $G$-isomorphic to $D(V)$, the element $R_{a}$ can be viewed as a $G$-invariant differential operator with polynomial coefficients. The operators $R_{a}$ are sometimes called Capelli operators. They are also called unnormalized canonical invariants in [Benson and Ratcliff 2004]. Moreover the family of elements $R_{\boldsymbol{a}}$ $\left(\boldsymbol{a} \in \mathbb{N}^{r+1}\right)$ is a vector basis of the vector space $D(V)^{G}=D(\mathcal{O})^{G}$.

The Capelli operators $R_{i}$ corresponding to the space $V_{\lambda_{i}}(i=0, \ldots, r)$ will be of particular importance because of the result below.

Theorem 2.2.9 (Howe and Umeda). Let $(G, V)$ be a MF space. The Capelli operators $R_{i}(i=0, \ldots, r)$ are algebraically independent and $D(V)^{G}=\mathbb{C}\left[R_{0}, \ldots, R_{r}\right]$.

Proof. See [Howe and Umeda 1991, Theorem 9.1; Benson and Ratcliff 2004, Corollary 7.4.4].

Remark 2.2.10. a) Recall that for $i=0,1, \ldots, k$ the polynomials $\Delta_{0}, \Delta_{1}, \ldots, \Delta_{k}$ are the fundamental relative invariants under the action of the full group $G$. Once these polynomials are fixed, let us define the polynomial $\Delta_{i}^{*} \in \mathbb{C}\left[V^{*}\right]$ as the unique fundamental relative invariant of $\left(G, V^{*}\right)$ with character $\lambda_{i}^{-1}$, such that $\Delta_{i}^{*}(\partial) \Delta_{i}(0)=1$, for $i=0, \ldots, k$. Then the Capelli operators $R_{i}(i=0, \ldots, k)$ are given by $R_{i}=\Delta_{i}(x) \Delta_{i}^{*}(\partial)$, and the Capelli operator corresponding to the irreducible component $V_{a_{0} \lambda_{0}+\cdots+a_{k} \lambda_{k}}$ is a scalar multiple of $\Delta_{0}^{a_{0}}(x) \cdots \Delta_{k}^{a_{k}}(x) \Delta_{0}^{*}(\partial)^{a_{0}} \cdots \Delta_{k}^{*}(\partial)^{a_{k}}$. More generally the Capelli operator $R_{a}$ corresponding to $V_{a}$, where

$$
\boldsymbol{a}=a_{0} \lambda_{0}+\cdots+a_{k} \lambda_{k}+a_{k+1} \lambda_{k+1}+\cdots+a_{r} \lambda_{r}
$$

is a scalar multiple of $\Delta_{0}^{a_{0}}(x) \cdots \Delta_{k}^{a_{k}}(x) R_{a_{k+1} \lambda_{k+1}+\cdots+a_{r} \lambda_{r}} \Delta_{0}^{*}(\partial)^{a_{0}} \cdots \Delta_{k}^{*}(\partial)^{a_{k}}$.

b) Moreover, in the case where $(G, V)$ is irreducible, as $\Delta_{r}$ is the highest weight vector in $V^{*}$, the operator $R_{r}$ is nothing but the Euler operator $E$.

c) More generally, if $V=V_{1} \oplus \cdots \oplus V_{\ell}$, where the representations $\left(G, V_{i}\right)$ are irreducible, the various Euler operators $E_{i}$ on $V_{i}$ are the Capelli operators associated to the irreducible subspaces $V_{i}^{*} \in \mathbb{C}[V]$. Of course the global Euler operator $E$ on $V$ is given by $E=E_{1}+\cdots+E_{\ell}$. As the highest weight vectors of the spaces $\left(G, V_{i}^{*}\right)$ occur as the last $\ell$ elements of $\Delta_{0}, \ldots, \Delta_{r}$, we have $R_{r-\ell+1}=E_{1}, \ldots, R_{r}=E_{\ell}$.

d) According to b) and c), one can always take $\left\{R_{0}, R_{1}, \ldots, R_{r-1}, E\right\}$ as a set of algebraically independent generators of $D(V)^{G}$. 
2.3. Multiplicity-free spaces with a one-dimensional quotient. Let us now define the main objects this paper deals with, namely the MF spaces with a one-dimensional quotient, which were introduced by T. Levasseur.

Definition 2.3.1 [Levasseur 2009, Sections 3.2 and 4.2]. 1) A prehomogeneous vector space $(G, V)$ is said to be of rank one* if there exists a homogeneous polynomial $\Delta_{0}$ on $V$ such that $\Delta_{0} \notin \mathbb{C}[V]^{G}$ and $\mathbb{C}[V]^{G^{\prime}}=\mathbb{C}\left[\Delta_{0}\right]$.

2) A multiplicity-free space $(G, V)$ is said to have a one-dimensional quotient if it is a PV of rank one.

Remark 2.3.2. a) The classification of multiplicity-free spaces with a one-dimensional quotient has been obtained in [Rubenthaler 2013].

b) It can be shown that if $(G, V)$ is a PV of rank one, then the polynomial $\Delta_{0}$ is the unique fundamental relative invariant of $(G, V)$. More precisely a $\mathrm{PV}(G, V)$ is of rank one if and only if it has a unique fundamental relative invariant [ibid.]. Hence in the notation of Section 2.2 we have $k=0$, in other words $\Delta_{0}$ is the unique fundamental $G$ relative invariant among the $B$ relative invariants $\Delta_{0}, \Delta_{1}, \ldots, \Delta_{r}$.

We give now some examples of MF spaces with a one-dimensional quotient.

Example 2.3.3. PVs of commutative parabolic type (for details we refer to [Muller et al. 1986]; [Rubenthaler and Schiffmann 1987] is also relevant).

Let $\tilde{\mathfrak{g}}$ be a simple complex Lie algebra. Assume we are given a 3-grading of $\tilde{\mathfrak{g}}$ :

$$
\tilde{\mathfrak{g}}=V^{-} \oplus \mathfrak{g} \oplus V^{+} .
$$

Then $\mathfrak{g}$ is a reductive Lie subalgebra and it is well known that the representation $\left(\mathfrak{g}, V^{+}\right)$is prehomogeneous (here $\mathfrak{g}$ acts on $V^{+}$via the bracket). Let $\widetilde{G}$ be the adjoint group of $\tilde{\mathfrak{g}}$ and let $G$ be the connected subgroup of $\widetilde{G}$ whose Lie algebra is $\mathfrak{g}$. Then the space $\left(G, V^{+}\right)$is multiplicity-free. Moreover such a space has a one-dimensional quotient if and only if it is a regular PV. Up to local isomorphism one obtains the following list:

1) $\left(\operatorname{SL}(n, \mathbb{C}) \times \operatorname{SL}(n, \mathbb{C}) \times \mathbb{C}^{*}, M_{n}(\mathbb{C})\right)$ acting via $\left(g_{1}, g_{2}, t\right) . x=\operatorname{tg}_{1} x g_{2}^{-1}$, where $g_{1}, g_{2} \in \operatorname{SL}(n, \mathbb{C}), t \in \mathbb{C}^{*}, x \in M_{n}(\mathbb{C})$; here $\Delta_{0}(x)=\operatorname{det}(x)$.

2) $\left(O(n, \mathbb{C}) \times \mathbb{C}^{*}, \mathbb{C}^{n}\right)$ with the natural action. Here $\Delta_{0}(x)=Q(x)=\sum_{i=1}^{i=n} x_{i}^{2}$.

3) (GL $(n, \mathbb{C}), \operatorname{Sym}_{n}(\mathbb{C})$ ), where $\operatorname{Sym}_{n}(\mathbb{C})$ denotes the $n \times n$ symmetric matrices, with the action $g \cdot x=g x^{t} g$. Then $\Delta_{0}(x)=\operatorname{det}(x)$.

4) $\left(\mathrm{GL}(n, \mathbb{C}), \operatorname{Skew}_{n}(\mathbb{C})\right), n$ even, with the action $g \cdot x=g x^{t} g$, where $\operatorname{Skew}_{n}(\mathbb{C})$ denotes the $n \times n$ skew-symmetric matrices. Then $\Delta_{0}(x)=\operatorname{Pf}(x)$, where $\operatorname{Pf}(x)$ denotes the pfaffian of the even skew-symmetric matrix $x$.

*If $(G, V)$ is also multiplicity-free, its rank as a PV is not the same as its rank as an MF space. 
5) $\left(E_{6} \times \mathbb{C}^{*}, \mathbb{C}^{27}\right)$ (the irreducible 27-dimensional representation of $\left.E_{6}\right)$. The fundamental relative invariant is of degree 3; it is known as the Freudenthal cubic.

Example 2.3.4. We consider $\left(\mathrm{GL}(2) \times \mathrm{Sp}(n), \mathbb{C}^{2} \otimes \mathbb{C}^{2 n}\right)$ (tensor product of the natural representations). Here the action is given by

$$
\left(g_{1}, g_{2}\right) . X=g_{2} X\left({ }^{t} g_{1}\right), \quad g_{1} \in \mathrm{SL}(2), g_{2} \in \mathrm{Sp}(n), X \in M_{2 n, 2}
$$

The relative invariant $\Delta_{0}$ is given by $\operatorname{Pf}\left({ }^{t} X J X\right)$, where

$$
J=\left(\begin{array}{cc}
0 & I d_{n} \\
-I d_{n} & 0
\end{array}\right)
$$

and where $P f(\cdot)$ is the pfaffian of a $2 \times 2$ skew symmetric matrix. The rank is equal to 3 and it is a regular PV. For details see [Howe and Umeda 1991, case 11.6; Rubenthaler 2013, case 4.1.7].

Example 2.3.5. (GL $\left.(n) \times \mathrm{GL}(n-1), M_{n, 1} \oplus M_{n, n-1}\right)$. The action is given by $\left(g_{1}, g_{2}\right)(v, x)=\left(g_{1} v, g_{1} x g_{2}^{-1}\right), g_{1} \in \mathrm{GL}(n), g_{2} \in \mathrm{GL}(n-1), v \in M_{n, 1}, x \in M_{n, n-1}$.

The relative invariant $\Delta_{0}$ is given by $\Delta_{0}(x)=\operatorname{det}(v ; x)$, where $(v ; x)$ is the $n \times n$ matrix obtained by putting the column vector $v$ left to the $n \times(n-1)$ matrix $x$. The rank is equal to $2 n-1$ and it is a regular PV. For details see [Benson and Ratcliff 2000, case 4.2.4; Rubenthaler 2013, case 4.2.5].

\section{Algebras of differential operators}

From now on we suppose that $(G, V)$ is an MF space with a one-dimensional quotient.

3.1. Gradings and Bernstein-Sato polynomials. Recall that $\Delta_{0}, \ldots, \Delta_{r}$ denote the fundamental relative invariants under a fixed Borel subgroup $B$ of $G$. As the space has a one-dimensional quotient, $\Delta_{0}$ is the unique polynomial among them which is relatively invariant under $G$ (this means that $k=0$ in the notation of Section 2.2). We also set $O=\left\{x \in V \mid \Delta_{0}(x) \neq 0\right\}$.

Of course the Euler operator $E$ on $V$, defined for $P \in \mathbb{C}[V]$ by

$$
E P(x)=\frac{\partial}{\partial t} P(t x)_{t=1}=P^{\prime}(x) x,
$$

is invariant by any element in $\operatorname{GL}(E)$.

Once and for all we also define the following two elements in $D(V)$ :

$$
\left.X=\Delta_{0} \text { (multiplication by } \Delta_{0}\right), \quad Y=\Delta_{0}^{*}(\partial) .
$$


The operator

$$
X^{-1} \text { (multiplication by } \Delta_{0}^{-1} \text { ), }
$$

which belongs to $D(0)$, will also play an important role. From the definition of the $G$ action on $\mathbb{C}[V]$ and on $D(V)$ we have

$$
g . X=\lambda_{0}\left(g^{-1}\right) X, \quad g . X^{-1}=\lambda_{0}(g) X^{-1}, \quad g . Y=\lambda_{0}(g) Y,
$$

and hence $X, Y \in D(V)^{G^{\prime}}$ and $X^{-1} \in D(\mathcal{O})^{G^{\prime}}$.

We now introduce some notation used in the rest of the paper:

$$
\mathscr{T}=D(\mathcal{O})^{G^{\prime}}, \quad \mathscr{T}_{0}=D(V)^{G}=D(\mathcal{O})^{G}
$$

(the last equality comes from Theorem 2.2.6). Remember that $\mathscr{T}_{0}$ is a polynomial algebra in $r+1$ variables (Theorem 2.2.9). We have the inclusions

$$
\mathscr{T}_{0}=D(V)^{G}=D(\mathbb{O})^{G} \subset D(V)^{G^{\prime}} \subset \mathcal{T}=D(\mathbb{O})^{G^{\prime}} .
$$

An element $D$ in $\mathscr{T}$ is said to be of degree $m$ if $[E, D]=m D$. As differential operators in $\mathscr{T}$ have coefficients which are fractions whose denominators are homogeneous (powers of $\Delta_{0}$ ), it is clear that $\mathscr{T}$ is graded by its homogeneous components. But on the other hand any homogeneous element $D$ in $\mathcal{T}$ preserves the $G^{\prime}$-isotypic components $W_{\tilde{\boldsymbol{a}}}=\bigoplus_{n \in \mathbb{N}} \Delta_{0}^{n} V_{\tilde{\boldsymbol{a}}}$ (see Proposition 2.2.8). Therefore a homogeneous element $D$ maps $\Delta_{0}^{n} V_{\tilde{\boldsymbol{a}}}$ on $\Delta_{0}^{n+j} V_{\tilde{\boldsymbol{a}}}$ for some $j$ and hence only multiples of $d_{0}$ (the degree of $\Delta_{0}$ ) occur as homogeneous degrees in $\mathscr{T}$. If we define, for $p \in \mathbb{Z}, \mathscr{T}_{p}=\left\{D \in \mathscr{T} \mid[E, D]=p d_{0} D\right\}$, then

$$
\mathscr{T}=\bigoplus_{p \in \mathbb{Z}} \mathscr{T}_{p}
$$

(At this point it is not completely evident that the two definitions of $\mathscr{T}_{0}$ coincide, that is, $D(V)^{G}=\{D \in \mathscr{T} \mid[E, D]=0\}$. This will be a consequence of the proof of Proposition 3.1.6 below.)

Similarly if we define

$$
D(V)_{p}^{G^{\prime}}=\left\{D \in D(V)^{G^{\prime}} \mid[E, D]=p d_{0} D\right\},
$$

we have $D(V)^{G^{\prime}}=\bigoplus_{p \in \mathbb{Z}} D(V)_{p}^{G^{\prime}}$.

Definition 3.1.1. For $\boldsymbol{a}=\left(a_{0}, a_{1}, \ldots, a_{r}\right)$ and $p \in \mathbb{N}$, we define

$$
\boldsymbol{a}+p=\left(a_{0}+p, a_{1}, \ldots, a_{r}\right) .
$$

Then if $D \in \mathscr{T}_{p}$, the Schur Lemma ensures that if $P \in V_{\boldsymbol{a}}$ we have $D P=b_{D}(\boldsymbol{a}) X^{p} P$, where $b_{D}(\boldsymbol{a}) \in \mathbb{C}$. It is easy to see that $b_{D}$ is a polynomial in the variables $\left(a_{0}, a_{1}, \ldots, a_{r}\right)$ (see for example [Knop 1998, proof of Corollary 4.4]). This polynomial is called the Bernstein-Sato polynomial of $D$. 
Example 3.1.2. Relations (3-1-1) imply that $X \in \mathscr{T}_{1}, X^{-1} \in \mathscr{T}_{-1}$ and $Y \in \mathscr{T}_{-1}$. And of course $E \in \mathscr{T}_{0}$. Obviously, from the definition, we have $b_{X}(\boldsymbol{a})=b_{X^{-1}}(\boldsymbol{a})=1$, $b_{E}(\boldsymbol{a})=d_{0} a_{0}+d_{1} a_{1}+\cdots+d_{r} a_{r}=$ the degree of $V_{\boldsymbol{a}}$ (recall that $d_{i}$ is the degree of $\Delta_{i}$ ). The computation of $b_{Y}$ is more difficult. However it is known in the case of PVs of commutative parabolic type (see Example 2.3.3). In this case, for $\boldsymbol{X}=\left(X_{0}, X_{1}, \ldots, X_{r}\right)$ it is given by

$$
b_{Y}(\boldsymbol{X})=c \prod_{j=0}^{r}\left(X_{0}+\cdots+X_{j}+j \frac{d}{2}\right),
$$

where the constant $c$ can be made explicit (see [Bopp and Rubenthaler 1993, Théorème 3.19]) and where $d / 2=\left(\operatorname{dim}(V)-d_{0}\right) /\left(\left(d_{0}-1\right) d_{0}\right)$. This explicit computation of the polynomial $b_{Y}$ in the particular case of PVs of commutative parabolic type has been obtained by several authors, using distinct methods (see [Kostant and Sahi 1991; Wallach 1992; Bopp and Rubenthaler 1993; Faraut and Korányi 1994]). The constant $d$ is the same as the constant $d$ which is familiar to specialists of Jordan algebras.

The following lemma is obvious, but useful.

Lemma 3.1.3. Let $D_{1}, D_{2} \in \mathscr{T}_{p}$. Then $D_{1}=D_{2}$ if and only if $b_{D_{1}}=b_{D_{2}}$.

Definition 3.1.4. The automorphism $\tau$ of $\mathscr{T}=D(\mathcal{O})^{G^{\prime}}$ is defined by

$$
\tau(D)=X D X^{-1} \text { for all } D \in \mathscr{T} .
$$

Proposition 3.1.5. The algebra $\mathscr{T}_{0}$ is stable under $\tau$ and for any $D \in \mathscr{T}_{0}$ we have

$$
\begin{gathered}
X D=\tau(D) X, \\
D Y=Y \tau(D) .
\end{gathered}
$$

Proof. By definition, $\mathscr{T}_{0}=D(V)^{G}$. From relations (3-1-1) we see that if $D$ is $G$-invariant so is $\tau(D)$. Obviously $\tau(D) \in D(\mathbb{O})^{G}$. But $D(\mathcal{O})^{G}=D(V)^{G}$ by Theorem 2.2.6; hence $\mathscr{T}_{0}$ is $\tau$-stable. Relation (3-1-4) is just the definition of $\tau$. We will now prove that (3-1-5) holds on each subspace $V_{\boldsymbol{a}}$. Let $b_{D}$ be the BernsteinSato polynomial of $D$. Then an easy calculation shows that the left and right sides of (3-1-5) act on $V_{\boldsymbol{a}}$ by $b_{D}(\boldsymbol{a}-1) b_{Y}(\boldsymbol{a}) X^{-1}$. Then Lemma 3.1 .3 implies (3-1-5).

Let us denote by $\mathscr{T}_{0}[X, Y]$ the subalgebra of $\mathscr{T}$ generated by $\mathscr{T}_{0}, X$ and $Y$. From the preceding proposition and from the fact that $X Y$ and $Y X$ belong to $\mathscr{T}_{0}$ we know that any element $D \in \mathscr{T}_{0}[X, Y]$ can be written as a finite sum $D=\sum_{p, q \in \mathbb{N}} a_{p, q} X^{p} Y^{q}$ with $a_{p, q} \in \mathscr{T}_{0}$. Similarly, let $\mathscr{T}_{0}\left[X, X^{-1}\right]$ denote the subalgebra of $\mathscr{T}$ generated by $\mathscr{T}_{0}, X$ and $X^{-1}$. Also any element $D$ in $\mathscr{T}_{0}\left[X, X^{-1}\right]$ can we written as a finite sum $D=\sum_{p \in \mathbb{Z}} a_{p} X^{p}$. The following proposition shows that $D(V)^{G^{\prime}}=\mathscr{T}_{0}[X, Y]$ and that $\mathscr{T}=D(\mathcal{O})^{G^{\prime}}=\mathscr{T}_{0}\left[X, X^{-1}\right]$ and makes the gradings more precise. 
Proposition 3.1.6. 1) We have

$$
D(V)^{G^{\prime}}=\mathscr{T}_{0}[X, Y]=\left(\bigoplus_{p \in \mathbb{N}^{*}} \mathscr{T}_{0} Y^{p}\right) \oplus \mathscr{T}_{0} \oplus\left(\bigoplus_{p \in \mathbb{N}^{*}} \mathscr{T}_{0} X^{p}\right)
$$

(in particular $D(V)_{p}^{G^{\prime}}=\mathscr{T}_{0} X^{p}$ if $p \geq 0$, and $D(V)_{p}^{G^{\prime}}=\mathscr{T}_{0} Y^{-p}$ if $p<0$ ). Equivalently,

$$
D(V)^{G^{\prime}}=\mathscr{T}_{0}[X, Y]=\left(\bigoplus_{p \in \mathbb{N}^{*}} Y^{p} \mathscr{T}_{0}\right) \oplus \mathscr{T}_{0} \oplus\left(\bigoplus_{p \in \mathbb{N}} X^{p} \mathscr{T}_{0}\right) .
$$

2) We have $\mathscr{T}=D(\mathcal{O})^{G^{\prime}}=\mathscr{T}_{0}\left[X, X^{-1}\right]=\bigoplus_{p \in \mathbb{Z}} \mathscr{T}_{0} X^{p}=\bigoplus_{p \in \mathbb{Z}} X^{p} \mathscr{T}_{0}$.

3) Any element $D$ in $\mathscr{T}_{0}[X, Y]$ can be written uniquely in the form

$$
D=\sum_{i>0} u_{i} Y^{i}+\sum_{i \geq 0} v_{i} X^{i} \quad \text { or } \quad D=\sum_{i>0} Y^{i} u_{i}+\sum_{i \geq 0} X^{i} v_{i} \quad \text { (finite sums) }
$$

with $u_{i}, v_{i} \in \mathscr{T}_{0}$.

Any element $D \in \mathcal{T}$ can be written uniquely in the form

$$
D=\sum_{i \in \mathbb{Z}} u_{i} X^{i} \quad \text { or } \quad D=\sum_{i \in \mathbb{Z}} X^{i} u_{i} \quad \text { (finite sums) }
$$

with $u_{i} \in \mathscr{T}_{0}$.

Proof. 1) For the moment we define $\mathscr{T}_{0}$ by $\mathscr{T}_{0}=D(V)^{G}$. From Proposition 2.2.8 we know that the decomposition of $\mathbb{C}[V]$ into $G^{\prime}$-isotypic components is given by

$$
\mathbb{C}[V]=\bigoplus_{\tilde{\boldsymbol{a}} \in \mathbb{N}^{r}} U_{\tilde{\boldsymbol{a}}}, \quad \text { where } U_{\tilde{\boldsymbol{a}}}=\bigoplus_{a_{0} \in \mathbb{N}} \Delta_{0}^{a_{0}} V_{\tilde{\boldsymbol{a}}} \text { and } \tilde{\boldsymbol{a}}=\left(0, a_{1}, \ldots, a_{r}\right) .
$$

We will now use the technique of [Howe and Umeda 1991] which we have already mentioned before Theorem 2.2.9. As $\mathbb{C}[V] \otimes \mathbb{C}\left[V^{*}\right]$ is $G^{\prime}$-isomorphic to $D(V)$, each subspace $\Delta^{a_{0}} V_{\tilde{\boldsymbol{a}}} \otimes\left(\Delta^{b_{0}} V_{\tilde{\boldsymbol{a}}}\right)^{*}$ will give rise to a unique $G^{\prime}$-invariant differential operator $R_{a_{0}, b_{0}, \tilde{\boldsymbol{a}}}$. Then by the same arguments as in Remark 2.2.10, it is easy to see that $R_{a_{0}, b_{0}, \tilde{\boldsymbol{a}}}=\Delta_{0}(x)^{a_{0}} R_{0,0, \tilde{\boldsymbol{a}}} \Delta_{0}^{*}(\partial)^{b_{0}}=X^{a_{0}} R_{0,0, \tilde{\boldsymbol{a}}} Y^{b_{0}}$. The elements $X^{a_{0}} R_{0,0, \tilde{\boldsymbol{a}}} Y^{b_{0}}\left(a_{0}, b_{0} \in \mathbb{N}, \tilde{\boldsymbol{a}} \in \mathbb{N}^{r}\right)$ form a vector basis of $D(V)^{G^{\prime}}$. Remark now that $R_{0,0, \tilde{a}}$ is in $D(V)^{G}=\mathscr{T}_{0}$. Then from Proposition 3.1.5, we get $X^{a_{0}} R_{0,0, \tilde{\boldsymbol{a}}} Y^{b_{0}}=\tau^{a_{0}}\left(R_{0,0, \tilde{\boldsymbol{a}}}\right) X^{a_{0}} Y^{b_{0}}$ and $\tau^{a_{0}}\left(R_{0,0, \tilde{\boldsymbol{a}}}\right) \in \mathscr{T}_{0}$. If now $a_{0} \leq b_{0}$, then $X^{a_{0}} R_{0,0, \tilde{\boldsymbol{a}}} Y^{b_{0}}=R Y^{b_{0}-a_{0}}$, where $R=\tau^{a_{0}}\left(R_{0,0, \tilde{\boldsymbol{a}}}\right) X^{a_{0}} Y^{a_{0}} \in \mathscr{T}_{0}$. If $a_{0}>b_{0}$, then $X^{a_{0}} R_{0,0, \tilde{\boldsymbol{a}}} Y^{b_{0}}=R X^{a_{0}-b_{0}}$, where $R=\tau^{a_{0}}\left(R_{0,0, \tilde{\boldsymbol{a}}}\right) \tau^{a_{0}-b_{0}}\left(X^{b_{0}} Y^{b_{0}}\right) \in \mathcal{T}_{0}$. The first decomposition in assertion 1$)$ is proved. The second decomposition is a consequence of relations (3-1-4) and (3-1-5).

2) A slight extension of (2-2-2) shows that $\mathbb{C}[\mathbb{O}] \otimes \mathbb{C}\left[V^{*}\right]$ is $G$-isomorphic to $D(\mathbb{O})$ through the map $\varphi \otimes f \mapsto \varphi f(\partial)$. Then the same proof as in 1) above shows that the 
elements $X^{a_{0}} R_{0,0, \tilde{\boldsymbol{a}}} Y^{b_{0}}\left(a_{0} \in \mathbb{Z}, b_{0} \in \mathbb{N}, \tilde{\boldsymbol{a}} \in \mathbb{N}^{r}\right)$ form a vector basis of $D(\mathcal{O})^{G^{\prime}}=\mathscr{T}$. Consider now an element $D \in \mathcal{T}$ such that $[E, D]=0$. Then necessarily $D$ is a linear combination of elements of the form $X^{a_{0}} R_{0,0, \tilde{a}} Y^{a_{0}}$ with $a_{0} \in \mathbb{N}$. Then, as announced previously, the two definitions of $\mathscr{T}_{0}$ coincide $\left(\mathscr{T}_{0}=D(V)^{G}\right.$ and $\left.\mathscr{T}_{0}=\{D \in \mathscr{T} \mid[E, D]=0\}\right)$. Now if $D \in \mathscr{T}_{p}$, then $D=D X^{-p} X^{p}$ and $D X^{-p} \in \mathscr{T}_{0}$. Hence $\mathscr{T}_{p}=\mathscr{T}_{0} X^{p}=X^{p} \mathscr{T}_{0}$.

Assertion 3) is then obvious.

Remark 3.1.7. The inclusion $D(V)^{G^{\prime}} \subset D(\mathbb{O})^{G^{\prime}}$ is obviously strict (note that $X^{-1} \in D(\mathcal{O})^{G^{\prime}} \backslash D(V)^{G^{\prime}}$ ), but the preceding results show that these two graded algebras have the same "positive part" $\left(\bigoplus_{p \in \mathbb{N}} \mathscr{T}_{0} X^{p}\right)$.

The following proposition, whose proof is straightforward, shows that all the Bernstein-Sato polynomials are known if one knows the Bernstein-Sato polynomials of $Y$ and of the elements of $\mathscr{T}_{0}$.

Proposition 3.1.8. Let $D=D_{0} X^{n}(n \in \mathbb{Z})$ and $D^{\prime}=D_{0} Y^{n}\left(n \in \mathbb{N}^{*}\right), D_{0} \in \mathscr{T}_{0}$, be generic homogeneous elements in $\mathscr{T}=\mathscr{T}_{0}\left[X, X^{-1}\right]$ and $\mathscr{T}_{0}[X, Y]$. Then $b_{D}(\boldsymbol{a})=$ $b_{D_{0}}(\boldsymbol{a}+n)$ and $b_{D^{\prime}}(\boldsymbol{a})=b_{D_{0}}(\boldsymbol{a}-n) b_{Y}(\boldsymbol{a}) b_{Y}(\boldsymbol{a}-1) \cdots b_{Y}(\boldsymbol{a}-n+1)$.

3.2. The Harish-Chandra isomorphism and the center of $\mathscr{T}$. The aim of this subsection is to describe $\mathscr{T}_{0}=D(V)^{G}$ as a module over the center of $\mathscr{T}$. For this we will use the Harish-Chandra isomorphism for MF spaces due to F. Knop.

Let $(G, V)$ be an MF space with a one-dimensional quotient. Let $B$ be a fixed Borel subgroup of $G$. Remember that $(B, V)$ is a PV. Recall also that we denote by $\Delta_{0}, \Delta_{1}, \ldots, \Delta_{r}$ the set of fundamental relative invariants of $(B, V)$ and that $\Delta_{0}$ is the unique fundamental relative invariant under $G$. We denote by $d_{i}\left(\operatorname{resp} . \lambda_{i}\right)$ the degree (resp. the infinitesimal character) of $\Delta_{i}$. Let $\mathfrak{b}$ be the Lie algebra of $B$, let $\mathfrak{t} \subset \mathfrak{b}$ be a Cartan subalgebra of $\mathfrak{g}$ and let $\Sigma$ be the set of roots of the pair ( $\mathfrak{g}, \mathfrak{t}$ ). Denote by $W$ the Weyl group of $\Sigma$. Denote by $\Sigma^{+}$the set of positive roots such that $\mathfrak{b}=\mathfrak{t}+\sum_{\alpha \in \Sigma^{+}} \mathfrak{g}^{\alpha}$. Let $\rho=\frac{1}{2} \sum_{\alpha \in \Sigma^{+}} \alpha$. We define

$$
\mathfrak{a}^{*}=\bigoplus_{i=0}^{r} \mathbb{C} \lambda_{i} \subset \mathfrak{t}^{*} \quad \text { and } \quad A=\mathfrak{a}^{*}+\rho \subset \mathfrak{t}^{*} .
$$

Let $\mathscr{L}(\mathfrak{g})$ be the center of the enveloping algebra of $\mathfrak{g}$. Denote by $\mathbb{C}\left[\mathfrak{t}^{*}\right]^{W}$ the $W$-invariant polynomials on $\mathfrak{t}^{*}$. One knows that the classical Harish-Chandra isomorphism is an isomorphism $H: \mathscr{L}(\mathfrak{g}) \rightarrow \mathbb{C}\left[\mathfrak{t}^{*}\right]^{W}$ which can be computed the following way. For any $\lambda \in \mathfrak{t}^{*}$, let $V_{\lambda}$ be the irreducible highest weight module with highest weight $\lambda$. It is well known that $\mathscr{L}(\mathfrak{g})$ acts by scalar multiplication on $V_{\lambda}$. The scalar by which an element $z \in \mathscr{L}(\mathfrak{g})$ acts on $V_{\lambda}$ is precisely $H(z)(\lambda+\rho)$.

The natural representation of $G$ on $\mathbb{C}[V]$ extends to a representation of the enveloping algebra $U(\mathfrak{g})$ on the same space $\mathbb{C}[V]$. Hence $z \in \mathscr{L}(\mathfrak{g})$ acts on $V_{\boldsymbol{a}}$ by the 
scalar $H(z)\left(-\lambda_{\boldsymbol{a}}+\rho\right),{ }^{\dagger}$ where $\lambda_{\boldsymbol{a}}=\sum_{i=0}^{r} a_{i} \lambda_{i}$ (remember that $\boldsymbol{a}=\left(a_{0}, \ldots, a_{r}\right)$ ). Conversely if $\lambda=a_{0} \lambda_{0}+\cdots+a_{r} \lambda_{r}$ we define $\boldsymbol{a}_{\lambda}=\left(a_{0}, \ldots, a_{r}\right) \in \mathbb{C}^{r+1}$. By abuse of notation if $b_{D}$ is the Bernstein-Sato polynomial of $D \in \mathscr{T}_{0}$, we set $b_{D}(\lambda)=b_{D}\left(\boldsymbol{a}_{\lambda}\right)$.

On the other hand any $D \in D(V)^{G}=\mathscr{T}_{0}$ acts on each $V_{\boldsymbol{a}}$ by the scalar $b_{D}(\boldsymbol{a})$, where $b_{D}(\boldsymbol{a})$ is the Bernstein-Sato polynomial of $D$. This allows us to define the map

$$
\begin{aligned}
h: D(V)^{G} & \rightarrow \mathbb{C}[A], \\
D & \mapsto h(D):-\lambda+\rho \mapsto h(D)(-\lambda+\rho)=b_{D}(\lambda),
\end{aligned}
$$

where $\mathbb{C}[A]$ denotes the algebra of polynomials on the affine space $A=\mathfrak{a}^{*}+\rho \subset \mathfrak{t}^{*}$.

Let $\pi(z)$ be the operator in $D(V)^{G}$ which represents the action of $z$ on $\mathbb{C}[V]$ and let $r: \mathbb{C}\left[\mathfrak{t}^{*}\right]^{W} \rightarrow \mathbb{C}[A]$ be the restriction homomorphism. It is clear from the definitions that the following diagram commutes:

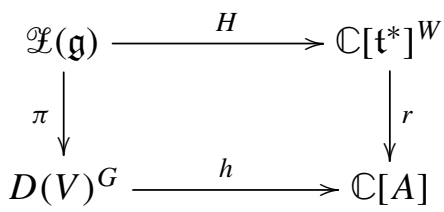

Theorem 3.2.1 [Knop 1998, Theorem 4.8 and Corollary 4.9; Benson and Ratcliff 2004, Theorem 9.2.1]. The homomorphism $h$ is injective and there exists a finite group $W_{0}$ (sometimes called the little Weyl group) which is a subgroup of the stabilizer of $A$ in $W$, such that the image of $h$ is $\mathbb{C}[A]^{W_{0}}$. Hence $h$ is an isomorphism between $D(V)^{G}$ and $\mathbb{C}[A]^{W_{0}}$. The isomorphism $h$ is called the Harish-Chandra isomorphism for the $M F$ space $(G, V)$. Moreover $W_{0}$ acts as a reflection group on $\mathfrak{a}^{*}$.

Let us see what is the automorphism of $\mathbb{C}[A]^{W_{0}}$ which corresponds to the action of $\tau$ on $D(V)^{G}$ through the Harish-Chandra isomorphism $h$. Let $D \in D(V)^{G}$. Then $h(\tau(D))(-\lambda+\rho)=h\left(X D X^{-1}\right)(-\lambda+\rho)=b_{X D X^{-1}}(\lambda)=b_{D}\left(\lambda-\lambda_{0}\right)$. This calculation proves of course that $\mathbb{C}[A]^{W_{0}}$ is stable under $P(\lambda+\rho) \mapsto P\left(\left(\lambda-\lambda_{0}\right)+\rho\right)$. Therefore we make the following definition.

Definition 3.2.2. By abuse of notation $\tau$ will also denote the automorphism of $\mathbb{C}[A]^{W_{0}}$ which is defined by $\tau(P)(\lambda+\rho)=P\left(\left(\lambda-\lambda_{0}\right)+\rho\right)\left(P \in \mathbb{C}[A]^{W_{0}}\right)$. Let $\mathbb{C}[A]^{W_{0}, \tau}$ denote the set of elements in $\mathbb{C}[A]^{W_{0}}$ that are invariant under $\tau$.

Proposition 3.2.3. Let $\mathscr{L}(\mathscr{T})$ be the center of $\mathscr{T}=D(\mathscr{O})^{G^{\prime}}$. Then $\mathscr{L}(\mathscr{T})$ is also the center of $\mathscr{T}_{0}[X, Y]=D(V)^{G^{\prime}}$. Moreover the following assertions are equivalent:

i) $D \in \mathscr{L}(\mathscr{T})$.

ii) $D \in \mathscr{T}_{0}$ and $\tau(D)=D$ (i.e., $D$ commutes with $X$ ).

${ }^{\dagger}$ The change of sign is due to the fact that we consider here characters of relative invariants instead of highest weights. 
iii) $D \in \mathscr{T}_{0}$ and the Bernstein-Sato polynomial $b_{D}\left(a_{0}, a_{1}, \ldots, a_{r}\right)$ does not depend on $a_{0}$.

iv) $D \in \mathscr{T}_{0}$ and $D$ commutes with $Y$.

v) $D \in \mathscr{T}_{0}$ and $h(D) \in \mathbb{C}[A]^{W_{0}, \tau}$.

Proof. i) $\Rightarrow$ ii): Let $D \in \mathscr{L}(\mathscr{T})$. Then $[E, D]=0$, hence $D \in \mathscr{T}_{0}$, and $[D, X]=0$.

ii) $\Rightarrow$ iii): Let $D \in \mathscr{T}_{0}$. If $X D=D X$ then, from the definitions we have

$$
\begin{aligned}
b_{X D}\left(a_{0}, a_{1}, \ldots, a_{r}\right) & =b_{D}\left(a_{0}, a_{1}, \ldots, a_{r}\right)=b_{D X}\left(a_{0}, a_{1}, \ldots, a_{r}\right) \\
& =b_{D}\left(a_{0}+1, a_{1}, \ldots, a_{r}\right)
\end{aligned}
$$

hence $b_{D}\left(a_{0}, a_{1}, \ldots, a_{r}\right)$ does not depend on $a_{0}$.

iii) $\Rightarrow$ i): Suppose that for $D \in \mathscr{T}_{0}$, the Bernstein-Sato polynomial does not depend on $a_{0}$. Then the elements $X D$ and $D X$ in $\mathscr{T}_{1}$ have the same Bernstein-Sato polynomial. Hence $X D=D X$ (Lemma 3.1.3). Then from Proposition 3.1.6(2) we see that $D \in \mathscr{L}(\mathscr{T})$.

iii) $\Rightarrow$ iv): Let $D \in \mathscr{T}_{0}$ such that $b_{D}$ does not depend on $a_{0}$. Then

$$
\begin{aligned}
b_{D Y}\left(a_{0}, a_{1}, \ldots, a_{r}\right) & =b_{D}\left(a_{0}-1, a_{1}, \ldots, a_{r}\right) b_{Y}\left(a_{0}, a_{1}, \ldots, a_{r}\right) \\
& =b_{D}\left(a_{0}, a_{1}, \ldots, a_{r}\right) b_{Y}\left(a_{0}, a_{1}, \ldots, a_{r}\right) \\
& =b_{Y D}\left(a_{0}, a_{1}, \ldots, a_{r}\right) .
\end{aligned}
$$

Hence $D Y=Y D$.

iv) $\Rightarrow$ iii): If $D Y=Y D$, then

$$
\begin{aligned}
b_{D Y}\left(a_{0}, a_{1}, \ldots, a_{r}\right) & =b_{D}\left(a_{0}-1, a_{1}, \ldots, a_{r}\right) b_{Y}\left(a_{0}, a_{1}, \ldots, a_{r}\right) \\
& =b_{Y D}\left(a_{0}, a_{1}, \ldots, a_{r}\right) \\
& =b_{Y}\left(a_{0}, a_{1}, \ldots, a_{r}\right) b_{D}\left(a_{0}, a_{1}, \ldots, a_{r}\right) .
\end{aligned}
$$

Hence $b_{D}$ does not depend on $a_{0}$.

The equivalence of iii) and v) is obvious since $h(D)(-\lambda+\rho)=b_{D}(\lambda)$.

From ii) we obtain that $\mathscr{L}(\mathscr{T})$ is also the center of $\mathscr{T}_{0}[X, Y]$.

Remark 3.2.4. As a consequence of the preceding proposition it is worthwhile noticing that if $D \in D(V)^{G^{\prime}}$ (or $D \in \mathscr{T}$ ) commutes with two operators among $(X, E, Y)$, then $D$ commutes with the third one. This is a well known property if $(X, E, Y)$ is an $\mathfrak{s l}_{2}$-triple. But we know from [Igusa 1981] that except if $\Delta_{0}$ is quadratic or linear the Lie algebra generated by $(X, E, Y)$ is infinite-dimensional. 
We will see in Theorem 4.2.2 that the associative algebra generated by $(X, E, Y)$ over $\mathscr{E}(\mathscr{T})$ is "similar" to $\mathscr{U}\left(\mathfrak{s l}_{2}(\mathscr{L}(\mathscr{T}))\right.$.

Define a linear form $\mu$ on $\mathfrak{a}^{*}$ by

$$
\mu\left(a_{0} \lambda_{0}+\cdots+a_{r} \lambda_{r}\right)=\sum_{i=0}^{r} a_{i} d_{i}=b_{E}(\boldsymbol{a}) \quad\left(\boldsymbol{a}=\left(a_{0}, \ldots, a_{r}\right) \in \mathbb{C}^{r+1}\right)
$$

( $\mu$ is the degree form, as its value on $\boldsymbol{a}=\left(a_{0}, \ldots, a_{r}\right) \in \mathbb{N}^{r+1}$ is equal to the degree of the polynomials in $V_{a}$ ). Define also

$$
\mathcal{M}=\left\{\lambda \in \mathfrak{a}^{*} \mid \mu(\lambda)=0\right\} \quad \text { and } \quad M=M+\rho \subset A .
$$

Note that $M=\{\lambda+\rho \in A \mid h(E)(-\lambda+\rho)=0\}$. As $h(E)$ is $W_{0}$-invariant, so is the set $M$. Set

$$
I(M)=\left\{P \in \mathbb{C}[A]^{W_{0}} \mid P_{\mid M}=0\right\} .
$$

The key lemma is the following.

Lemma 3.2.5. We have $I(M)=\mathbb{C}[A]^{W_{0}} h(E)$ and

$$
\mathbb{C}[A]^{W_{0}}=\mathbb{C}[A]^{W_{0}, \tau} \oplus I(M) .
$$

Proof. Let $P \in I(M)$. Then $P$ is a polynomial on the affine subspace $A \subset \mathfrak{t}^{*}$ vanishing on $M$, the set of zeros of the irreducible polynomial $h(E)$. Therefore $P=h(E) Q$. As $P$ and $h(E)$ are $W_{0}$-invariant, so is also the polynomial $Q$. Hence $I(M) \subset \mathbb{C}[A]^{W_{0}} h(E)$. The reverse inclusion is obvious.

Let $F=\mathbb{C} \lambda_{0} \subset \mathfrak{a}^{*}$. As obviously $\mathfrak{a}^{*}=M \oplus F$, we have $A=M \oplus F$. Remember that $\mathfrak{t}=\mathfrak{c} \oplus \mathfrak{t}^{\prime}$, where $\mathfrak{c}$ is the center of $\mathfrak{g}$. The infinitesimal character $\lambda_{0}$ is a character of $\mathfrak{g}$, and is therefore trivial on $\mathfrak{t}^{\prime} \subset \mathfrak{g}^{\prime}$. As any $w_{0} \in W_{0}$ fixes pointwise the center $\mathfrak{c}$ of $\mathfrak{g}$, we see that $F$ is pointwise fixed by $W_{0}$.

Let $Q \in \mathbb{C}[M]^{W_{0}}$. Define

$$
\widetilde{Q}(m+f)=Q(m), \quad \text { for all } m \in M, f \in F .
$$

From the preceding discussion we obtain that $\widetilde{Q}$ is $W_{0}$-invariant; in other words $\widetilde{Q} \in \mathbb{C}[A]^{W_{0}}$. But in fact $\widetilde{Q}$ is also $\tau$-invariant:

$$
\tau(\widetilde{Q})(m+f)=\widetilde{Q}\left(m+f-\lambda_{0}\right)=Q(m)=\widetilde{Q}(m+f) .
$$

Hence $\widetilde{Q} \in \mathbb{C}[A]^{W_{0}, \tau}$; in other words any $W_{0}$-invariant polynomial on $M$ can be extended to a $\left(W_{0}, \tau\right)$-invariant polynomial on $A$. This extension is in fact unique: for any $\tau$-invariant extension $\widetilde{\widetilde{Q}}$ of $Q$ we have $\widetilde{\widetilde{Q}}\left(m+x \lambda_{0}\right)=\widetilde{\widetilde{Q}}\left(m+(x+1) \lambda_{0}\right)$ 
and hence $\widetilde{Q}=\widetilde{Q}$. Hence we have proved that the restriction map

$$
\begin{aligned}
\mathbb{C}[A]^{W_{0}, \tau} & \rightarrow \mathbb{C}[M]^{W_{0}}, \\
P & \mapsto P_{\mid M},
\end{aligned}
$$

is bijective (and therefore $\mathbb{C}[A]^{W_{0}, \tau} \cap I(M)=\{0\}$ ) and the inverse map is $Q \mapsto \widetilde{Q}$. Now for $P \in \mathbb{C}[A]^{W_{0}}$ we can write

$$
P=\widetilde{P_{\mid M}}+\left(P-\widetilde{P_{\mid M}}\right) .
$$

From the discussion above we have $\widetilde{P_{\mid M}} \in \mathbb{C}[A]^{W_{0}, \tau}$, and $\left(P-\widetilde{P_{\mid M}}\right) \in I(M)$.

Theorem 3.2.6. 1) $\mathscr{T}_{0}=D(V)^{G}=\mathscr{L}(\mathscr{T}) \oplus E \mathscr{T}_{0}$.

2) Any element $H \in D(V)^{G}$ can be uniquely written in the form

$$
H=H_{0}+E H_{1}+E^{2} H_{2}+\cdots+E^{k} H_{k},
$$

where $H_{i} \in \mathscr{L}(\mathscr{T}), i=1,2, \ldots, k \in \mathbb{N}$.

Proof. Through the Harish-Chandra isomorphism $h$, the algebra $D(V)^{G}=\mathscr{T}_{0}$ corresponds to $\mathbb{C}[A]^{W_{0}}$, the algebra $\mathscr{L}(\mathscr{T})$ corresponds to $\mathbb{C}[A]^{W_{0}, \tau}$ and the ideal $E \mathscr{T}_{0}$ corresponds to $I(M)$. Therefore the first assertion is just the pullback by $h$ of the decomposition obtained in Lemma 3.2.5.

An element $H \in D(V)^{G}$ can therefore be uniquely written $H=H_{0}+E H^{1}$, with $H_{0} \in \mathscr{L}(\mathscr{T})$, and $H^{1} \in \mathscr{T}_{0}$. By induction we obtain a decomposition

$$
H=H_{0}+E H_{1}+E^{2} H_{2}+\cdots+E^{k-1} H_{k-1}+E^{k} H^{k},
$$

where $H_{0}, \ldots, H_{k-1} \in \mathscr{L}(\mathscr{T})$, and $H^{k} \in \mathscr{T}_{0}$. The process stops because if $k$ is greater than the degree in $a_{0}$ of $b_{H}$, then necessarily $H^{k}=0$ (see Proposition 3.2.3).

From this theorem and Proposition 3.1.6 we obtain immediately this consequence:

Corollary 3.2.7. 1) Let $D \in \mathcal{T}$. Then $D$ can be written uniquely in the form

$$
D=\sum_{\substack{k \in \mathbb{Z} \\ \ell \in \mathbb{N}}} H_{k, \ell} E^{\ell} X^{k} \quad \text { or } \quad D=\sum_{\substack{k \in \mathbb{\mathbb { N }} \\ \ell \in \mathbb{N}}} H_{k, \ell} X^{k} E^{\ell} \quad \text { (finite sums), }
$$

where $H_{k, \ell} \in \mathscr{L}(\mathscr{T})$.

2) Let $D \in \mathscr{T}_{0}[X, Y]$. Then $D$ can be written uniquely in the form

$$
\begin{aligned}
& D=\sum_{\substack{k \in \mathbb{N}^{*} \\
\ell \in \mathbb{N}}} H_{k, \ell} E^{\ell} Y^{k}+\sum_{\substack{r \in \mathbb{N} \\
s \in \mathbb{N}}} H_{r, s}^{\prime} E^{s} X^{r} \quad \text { (finite sums) or } \\
& D=\sum_{\substack{k \in \mathbb{N}^{*} \\
\ell \in \mathbb{N}}} H_{k, \ell} Y^{k} E^{\ell}+\sum_{\substack{r \in \mathbb{N} \\
s \in \mathbb{N}}} H_{r, s}^{\prime} X^{r} E^{s} \quad \text { (finite sums), }
\end{aligned}
$$

where $H_{k, \ell}, H_{r, s}^{\prime} \in \mathscr{L}(\mathscr{T})$. 
Corollary 3.2.8. Let $P \in \mathbb{C}[A]^{W_{0}}$. Then $P$ can be uniquely written in the form

$$
P(-\lambda+\rho)=\sum_{i=0}^{p} \alpha_{i}(-\lambda+\rho)\left(a_{0} d_{0}+a_{1} d_{1}+\cdots+a_{r} d_{r}\right)^{i},
$$

where $\alpha_{i} \in \mathbb{C}[A]^{W_{0}, \tau}$ and $\lambda=a_{0} \lambda_{0}+a_{1} \lambda_{1}+\cdots+a_{r} \lambda_{r} \in \mathfrak{a}^{*}$.

Proof. As $h(E)(-\lambda+\rho)=a_{0} d_{0}+a_{1} d_{1}+\cdots+a_{r} d_{r}$, the preceding decomposition is just the image through the Harish-Chandra isomorphism of the decomposition in Theorem 3.2.6(2).

Remark 3.2.9. It is easy to see that as $W_{0}$ stabilizes the affine space $A=\mathfrak{a}^{*}+\rho$ it also stabilizes $\mathfrak{a}^{*}$ (this is implicit in Theorem 3.2.1). Moreover if we denote by $0_{\rho}$ the barycenter of the $W_{0}$-orbit of $\rho$, then $0_{\rho}$ is a fixed point of the $W_{0}$-action on $A$ which is in $M$. As $\mathbb{C}[A]^{W_{0}}=\mathbb{C}\left[\mathfrak{a}^{*}+\rho\right]^{W_{0}}=\mathbb{C}\left[\mathfrak{a}^{*}+0_{\rho}\right]^{W_{0}} \simeq \mathbb{C}\left[\mathfrak{a}^{*}\right]^{W_{0}}$, and as $\mathscr{T}_{0}=D(V)^{G} \simeq \mathbb{C}[A]^{W_{0}}$ is a polynomial algebra in $r+1$ variables by Theorem 2.2.9, the group $W_{0}$ acts as a reflection group on $\mathfrak{a}^{*}$ by the Shephard-Todd-Chevalley theorem (this is a part of Knop's argument for Theorem 3.2.1). Hence by the theorem of Chevalley, the $r+1$ algebraically independent generators of the algebra $\mathbb{C}[A]^{W_{0}} \simeq \mathbb{C}\left[\mathfrak{a}^{*}\right]^{W_{0}}$ can be chosen to be homogeneous, either as functions on the vector space $\mathfrak{a}^{*}$, or as functions on $A$, for the vector space structure on $A$ defined by taking $0_{\rho}$ as origin.

We will now describe more precisely the algebra $\mathscr{L}(\mathscr{T})$.

Theorem 3.2.10. 1) $\mathscr{L}(\mathscr{T})$ is a polynomial algebra in $r$ variables. For $D \in \mathscr{T}_{0}$, let us denote by $\bar{D}$ the projection of $D$ on $\mathscr{L}(\mathscr{T})$ according to the decomposition $\mathscr{T}_{0}=\mathscr{L}(\mathscr{T}) \oplus E \mathscr{T}_{0}$. Remember from Theorem 2.2.9 that the set $R_{0}, \ldots, R_{r-1}, R_{r}$ of Capelli operators associated to the invariants $\Delta_{0}, \Delta_{1}, \ldots, \Delta_{r}$ ordered by decreasing degree is a set algebraically independent generators of $\mathcal{T}_{0}$. Then $\left\{\overline{R_{0}}, \ldots, \overline{R_{r-1}}\right\}$ is a set of algebraically independent generators of $\mathscr{L}(\mathscr{T})$.

2) Let $D$ be an element of $\mathscr{T}_{0}$ and let $b_{D}$ be its Bernstein-Sato polynomial. Then the Bernstein-Sato polynomial of $\bar{D}$ is given by

$$
b_{\bar{D}}\left(a_{0}, a_{1}, \ldots, a_{r}\right)=b_{D}\left(-\frac{a_{1} d_{1}+\cdots+a_{r} d_{r}}{d_{0}}, a_{1}, \ldots, a_{r}\right) .
$$

Proof. 1) Let us remark first that $\mathscr{L}(\mathscr{T})$ is already known to be a polynomial algebra from a result of Knop [1994]. He has proved that for a regular action of a reductive group on a smooth affine variety the center of the ring of invariant differential operators is always a polynomial algebra. We give here a direct proof and obtain some extra information. We know from Proposition 3.2.3 that $\mathscr{L}(\mathscr{T})$ is isomorphic, through the Harish-Chandra isomorphism $h$, to $\mathbb{C}[A]^{W_{0}, \tau}$. From the proof of Lemma 3.2.5 we know that $W_{0}$ stabilizes $M$ and that $\mathbb{C}[A]^{W_{0}, \tau} \simeq \mathbb{C}[M]^{W_{0}}=\left(\mathbb{C}[A]^{W_{0}}\right)_{\left.\right|_{M}}$. As 
$W_{0}$ is a reflection group on $A$ (this means that it is generated by the reflections it contains), so is $W_{\left.0\right|_{M}}$. Therefore $\mathbb{C}[M]^{W_{0}}$ (and hence $\mathscr{L}(\mathscr{T})$ ) is a polynomial algebra in $r=\operatorname{dim} M$ variables by Chevalley's Theorem. We know from Remark 2.2.10(d) that $\left\{R_{0}, \ldots, R_{r-1}, E\right\}$ is also a set algebraically independent generators of $\mathscr{T}_{0}$; hence $\left\{h\left(R_{0}\right), \ldots, h\left(R_{r-1}\right), h(E)\right\}$ is a set of algebraically independent generators of $\mathbb{C}[A]^{W_{0}}$. We obtain that $\mathbb{C}[M]^{W_{0}}=\mathbb{C}\left[h\left(R_{0}\right)_{\left.\right|_{M}}, \ldots, h\left(R_{r-1}\right)_{\left.\right|_{M}}\right]$ as $h(E)_{\mid M}=0$. As the transcendence degree of $\operatorname{Frac}\left(\mathbb{C}[M]^{W_{0}}\right)$ over $\mathbb{C}$ is $r$, the generators $h\left(R_{0}\right)_{\left.\right|_{M}}, \ldots, h\left(R_{r-1}\right)_{\mid M}$ are algebraically independent. Taking their inverse image under $h$ gives the first assertion of the theorem.

2) As we have seen the decomposition $\mathscr{T}_{0}=\mathscr{L}(\mathscr{T}) \oplus E \mathscr{T}_{0}$ is nothing else but the inverse image under $h$ of the decomposition $\mathbb{C}[A]^{W_{0}}=\mathbb{C}[A]^{W_{0}, \tau} \oplus I(M)$. Let $D \in \mathscr{T}_{0}$. From the proof of Lemma 3.2.5 we have $h(\bar{D})=\widetilde{h(D)_{\mid M}}$, where $\widetilde{h(D)_{\mid M}}$ is the unique $\left(W_{0}, \tau\right)$-invariant extension to $A$ of $h(D)_{\mid M}$. For $\lambda=a_{0} \lambda_{0}+\cdots+a_{r} \lambda_{r} \in \mathfrak{a}^{*}$, we have $h(E)(\lambda+\rho)=b_{E}(-\lambda)=-\left(a_{0} d_{0}+\cdots+a_{r} d_{r}\right)=-\mu(\lambda)$ (the degree form). Remember also that $\mathfrak{a}^{*}=\mathcal{M} \oplus$, where $\mu=\operatorname{ker}(\mu)$ and $F=\mathbb{C} \lambda_{0}$. Let us write $\lambda=m_{\lambda}+\alpha \lambda_{0}$, according to this decomposition. Then $b_{E}(\lambda)=\alpha b_{E}\left(\lambda_{0}\right)=\alpha d_{0}$. Hence $\alpha=\mu(\lambda) / d_{0}$ and $m_{\lambda}=\lambda-\left(\mu(\lambda) / d_{0}\right) \lambda_{0}$. Then we obtain

$$
\begin{aligned}
b_{\bar{D}}(\lambda) & =h(\bar{D})(-\lambda+\rho)=\widehat{h(D)_{\mid M}}(-\lambda+\rho) \\
& =\widehat{h(D)_{\mid M}}\left(-\lambda+\frac{\mu(\lambda)}{d_{0}} \lambda_{0}-\frac{\mu(\lambda)}{d_{0}} \lambda_{0}+\rho\right)=\widehat{h(D)_{\mid M}}\left(-\lambda+\frac{\mu(\lambda)}{d_{0}} \lambda_{0}+\rho\right) \\
& =h(D)_{\mid M}\left(-\lambda+\frac{\mu(\lambda)}{d_{0}} \lambda_{0}+\rho\right)=h(D)\left(-\lambda+\frac{\mu(\lambda)}{d_{0}} \lambda_{0}+\rho\right) \\
& =b_{D}\left(\lambda-\frac{\mu(\lambda)}{d_{0}} \lambda_{0}\right) .
\end{aligned}
$$

If we translate this into the $\left(a_{0}, \ldots, a_{r}\right)$-variables we obtain the second assertion.

Corollary 3.2.11. Let $b_{Y}$ be the Bernstein-Sato operator of $Y$. For any $\ell \in \mathbb{N}$ the element of $\operatorname{End}(\mathbb{C}[V])$ that acts on each space $V_{a}$ as scalar multiplication by $b_{Y}\left(-\left(a_{1} d_{1}+\cdots+a_{r} d_{r}\right) / d_{0}+\ell, a_{1}, \ldots, a_{r}\right)$ is the differential operator

$$
\overline{X^{1-\ell} Y X^{\ell}} \in \mathscr{L}(\mathscr{T}) \text {. }
$$

Moreover, if $\left(G, V^{+}\right)$is a PV of commutative parabolic type, the differential operators $\overline{X^{1-\ell} Y X^{\ell}}(\ell=0,1, \ldots, r)$ are generators of $\mathscr{E}(\mathscr{T})$.

Proof. As $b_{X^{1-\ell} Y X^{\ell}}\left(a_{0}, \ldots, a_{r}\right)=b_{Y}\left(a_{0}+\ell, a_{1}, \ldots, a_{r}\right)$, the first assertion follows immediately from Theorem 3.2.10. If $\left(G, V^{+}\right)$is a PV of commutative parabolic type, we know from Theorem 3.3.1 below that the operators $X^{1-\ell} Y X^{\ell}(\ell=0, \ldots, r)$ are (algebraically independent) generators of $\mathscr{T}_{0}$. 
3.3. The case of regular $P V$ 's of commutative parabolic type. In the case where $\left(G, V^{+}\right)$is a regular PV of commutative parabolic type (see Example 2.3.3), we obtain some specific results.

Theorem 3.3.1. Let $\left(G, V^{+}\right)$be a regular PV of commutative parabolic type.

1) The degree of $\Delta_{0}$ is equal to $r+1$ which is the rank of $\left(G, V^{+}\right)$as a MF space. More generally the degree of $\Delta_{i}$ is equal to $r+1-i$.

2) For $\ell \in \mathbb{Z}$ set $D_{\ell}=X^{1-\ell} Y X^{\ell}$. Then $D_{0}, D_{1}, \ldots, D_{r}$ are algebraically independent generators of $\mathscr{T}_{0}=D\left(V^{+}\right)^{G}$ (i.e., $\mathscr{T}_{0}=\mathbb{C}\left[D_{0}, D_{1}, \ldots, D_{r}\right]$ ).

3) We have $\mathcal{T}=D\left(\Omega^{+}\right)^{G^{\prime}}=\mathbb{C}\left[X, X^{-1}, Y\right]$, where $\mathbb{C}\left[X, X^{-1}, Y\right]$ is the associative subalgebra of $D\left(\Omega^{+}\right)$generated by $X, X^{-1}, Y$.

4) We have $\mathscr{T}_{0}[X, Y]=D(V)^{G^{\prime}}=\mathbb{C}\left[X, Y, R_{1}, \ldots, R_{r}\right]$, where the $R_{i}$ are the Capelli operators introduced before Theorem 2.2.9 and $\mathbb{C}\left[X, Y, R_{1}, \ldots, R_{r}\right]$ is the associative subalgebra of $D\left(V^{+}\right)$generated by $X, Y, R_{1}, \ldots, R_{r}$.

Proof. 1) This first assertion is proved in [Muller et al. 1986, Proposition 2.16 and Lemme 3.7].

2) We need now to use some technical results from the structure theory of commutative PVs of parabolic type. For details see [Muller et al. 1986; Rubenthaler and Schiffmann 1987]. We need also results concerning the symmetric space structure of the open $G$ orbit $\Omega^{+}$in $V^{+}$; they can be found in [Bopp and Rubenthaler 1993]. Let $\mathfrak{t}$ be a Cartan subalgebra of $\mathfrak{g}$; then $\mathfrak{t}$ is also a Cartan subalgebra of $\tilde{\mathfrak{g}}$ (see the notation in Example 2.3.3). Let $\widetilde{\Sigma}$ and $\Sigma$ be the root systems of $(\tilde{\mathfrak{g}}, \mathfrak{t})$ and $(\mathfrak{g}, \mathfrak{t})$, respectively. We choose an order on $\widetilde{\Sigma}$ such that the roots occurring in $V^{+}$are positive. We know from Proposition 2.9. in [Bopp and Rubenthaler 1993] that the open $G$-orbit $\Omega^{+}=\left\{x \in V^{+} \mid \Delta_{0}(x) \neq 0\right\}$ is a symmetric space $G / H$, where $H$ is the isotropy subgroup of a point $I^{+} \in \Omega^{+}$. The choice of $I^{+}$can be made the following way. It is known that any maximal set of strongly orthogonal long roots occurring in $V^{+}$has $r+1=\operatorname{rank}\left(G, V^{+}\right)$elements. There is a canonical way to construct such a maximal set, called the "descent"; see [Muller et al. 1986, Theorem 2.7, p. 101]. If $\left\{\alpha_{0}, \alpha_{1}, \ldots, \alpha_{r}\right\}$ is such a maximal set of strongly orthogonal long roots, then the element $I^{+}=X_{\alpha_{0}}+X_{\alpha_{1}}+\cdots+X_{\alpha_{r}}$ is generic (here as usual the $X_{\alpha_{i}}$ are nonzero root vectors). Let $\mathfrak{h}=Z_{\mathfrak{g}}\left(I^{+}\right)$be the Lie algebra of $H$, and let $\mathfrak{q}$ be the orthogonal complement of $\mathfrak{h}$ in $\mathfrak{g}$ with respect to the Killing form of $\tilde{\mathfrak{g}}$. Let $H_{\alpha_{i}} \in \mathfrak{t}$ be the coroot of $\alpha_{i}$. Set $\mathfrak{a}=\sum_{i=0}^{r} \mathbb{C} H_{\alpha_{i}}$. Then $\mathfrak{a}$ is a maximal abelian subspace of $\mathfrak{q}$ [Bopp and Rubenthaler 1993, Proposition 5.4] and the dual space $\mathfrak{a}^{*}$ can be identified with the space of restrictions of the fundamental characters $\lambda_{0}, \lambda_{1}, \ldots, \lambda_{r}$ [ibid., Lemme 2.5]. Hence this definition of $\mathfrak{a}^{*}$ is coherent with the direct definition of $\mathfrak{a}^{*}$ given in Section 3.2 in the general case $\left(\mathfrak{a}^{*}=\sum_{i=0}^{r} \mathbb{C} \lambda_{i}\right)$. 
For $\lambda \in \mathfrak{t}^{*}$, we will denote by $\bar{\lambda}$ the restriction of $\lambda$ to $\mathfrak{a}$. Through the "classical" Harish-Chandra isomorphism $\gamma$ for symmetric spaces [Heckman and Schlichtkrull 1994, Part II, Theorem 4.3] the algebra $\mathscr{T}_{0}$ is isomorphic to $S(\mathfrak{a})^{W_{R}}=\mathbb{C}\left[\mathfrak{a}^{*}\right]^{W_{R}}$, where $W_{R}$ is the Weyl group of the root system $R$ of $(\mathfrak{g}, \mathfrak{a})$. This root system is known to be of type $A_{r}$ (the proof is the same as for Theorem 3.11 in [Bopp and Rubenthaler 2005]). Hence $W_{R}$ is the symmetric group of $r+1$ variables and it acts by permutations on the $\bar{\alpha}_{i}$. We will choose an order on $R$ such that $\overline{\Sigma^{+}} \subset R^{+}$. As in [Muller et al. 1986; Rubenthaler and Schiffmann 1987] we consider here relative invariants $\Delta_{0}, \Delta_{1}, \ldots, \Delta_{r}$ with respect to the Borel subgroup defined by $\Sigma^{-}$. Define $\rho=\frac{1}{2} \sum_{\beta \in R^{-}} \beta$. It is well known that for $D \in \mathscr{T}_{0}$ and $\lambda=\sum_{i=0}^{r} a_{i} \lambda_{i} \in \mathfrak{a}^{*}, \gamma(D)(-\bar{\lambda}+\rho)$ is equal to the eigenvalue of $D$ acting on $\Delta_{0}^{a_{0}} \cdots \Delta_{r}^{a_{r}}$. In other words we have

$$
\gamma(D)(-\bar{\lambda}+\rho)=b_{D}(\lambda) .
$$

From [Rubenthaler and Schiffmann 1990, Lemme 3.9, p. 155], we know that

$$
\rho=\frac{d}{4} \sum_{i<j}\left(\bar{\alpha}_{i}-\bar{\alpha}_{j}\right)=\frac{d}{4} \sum_{i=0}^{r}(r-2 i) \bar{\alpha}_{i}
$$

and from [ibid., Lemme 3.8, p. 155], we also have

$$
\bar{\lambda}=a_{0} \bar{\alpha}_{0}+\left(a_{0}+a_{1}\right) \bar{\alpha}_{1}+\cdots+\left(a_{0}+\cdots+a_{r}\right) \bar{\alpha}_{r}
$$

Let us now make the following change of variables:

$$
s_{i}=a_{0}+\cdots+a_{i} \text {, for } i=0, \ldots, r .
$$

As $b_{D_{\ell}}(\lambda)=b_{Y}\left(s_{0}+\ell, \ldots, s_{r}+\ell\right)=c \prod_{i=0}^{r}\left(s_{i}+\ell+i d / 2\right)$ (see Example 3.1.2) we obtain

$$
\begin{aligned}
\gamma\left(D_{\ell}\right)(\bar{\lambda}) & =b_{D_{\ell}}(-\lambda+\rho)=b_{D_{\ell}}\left(\sum_{i=0}^{r}-s_{i} \bar{\alpha}_{i}+\frac{d}{4} \sum_{i=0}^{r}(r-2 i) \bar{\alpha}_{i}\right) \\
& =c \prod_{i=0}^{r}\left(-s_{i}+\frac{d}{4} r+\ell\right) .
\end{aligned}
$$

As expected the polynomials $\gamma\left(D_{\ell}\right)$ are symmetric in the $s_{i}$ variables (i.e., invariant under $W_{R}$ ). Moreover it is easy to prove that these polynomials, for $\ell=0, \ldots, r$, are algebraically independent generators of the algebra of symmetric polynomials. This proves 2 ).

\$The change of sign with respect to Lemme 3.8 in [Rubenthaler and Schiffmann 1990] is again due to the fact that we consider here characters of relative invariants instead of the highest weights. 
3) As $\mathscr{T}=\mathscr{T}_{0}\left[X, X^{-1}\right]$ (see Proposition 3.1.6), and as, from 2), the elements of $\mathscr{T}_{0}$ are polynomials in $X, X^{-1}, Y$ we obtain that $\mathscr{T} \subset \mathbb{C}\left[X, X^{-1}, Y\right]$. The inverse inclusion is obvious.

4) The inclusion $\mathbb{C}\left[X, Y, R_{1}, \ldots, R_{r}\right] \subset D\left(V^{+}\right)^{G^{\prime}}=\mathscr{T}_{0}[X, Y]$ is obvious. Conversely, from Theorem 2.2.9 we have $\mathscr{T}_{0}[X, Y]=\mathbb{C}\left[R_{0}, R_{1}, \ldots, R_{r}\right][X, Y]$. As $R_{0}=X Y$ (see Remark 2.2.10), we have $\mathscr{T}_{0}[X, Y] \subset \mathbb{C}\left[X, Y, R_{1}, \ldots, R_{r}\right]$.

Remark 3.3.2. According to [Terras 1988, p. 208], the operators $D_{\ell}$ were first considered by Selberg on positive definite symmetric matrices. They appear also in [Maaß 1971], in the same context of positive definite symmetric matrices. In the setting of symmetric cones, the analogue of assertion 2) of the preceding theorem can be found in [Faraut and Korányi 1994, Corollary XIV.1.6].

Remark 3.3.3. Note that for PVs of commutative parabolic type we have $R_{r}=E$. In the special case where $G \simeq \mathrm{SO}(k) \times \mathbb{C}^{*}$ and $V^{+} \simeq \mathbb{C}^{k}$, we have always $r=1$, and assertion 4 ) of the preceding theorem yields

$$
D\left(\mathbb{C}^{k}\right)^{\mathrm{SO}(k)}=\mathbb{C}[Q(x), Q(\partial), E],
$$

where $Q(x)=X=\sum_{i=1}^{k} x_{i}^{2}, Q(\partial)=Y=\sum_{i=1}^{k} \partial^{2} / \partial x_{i}^{2}$.

This was proved by S. Rallis and G. Schiffmann [1980, Lemma 5.2, p. 112].

\section{The structure of $D(V)^{G^{\prime}}$}

4.1. Smith algebras over rings. As usual if $a, b$ are elements of an associative algebra we define $[a, b]=a b-b a$.

Definition 4.1.1. Let $\boldsymbol{A}$ be a commutative associative algebra over $\mathbb{C}$, with unit element 1 and without zero divisors. Let $f, u \in A[t]$ be two polynomials in one variable with coefficients in $\boldsymbol{A}$. Let $n \in \mathbb{N}^{*}$.

1) The Smith algebra $S(\boldsymbol{A}, f, n)$ is the associative algebra over $\boldsymbol{A}$ with generators $(x, y, e)$ subject to the relations $[e, x]=n x,[e, y]=-n y,[y, x]=f(e)$.

2) The algebra $U(\boldsymbol{A}, u, n)$ is the associative algebra over $\boldsymbol{A}$ with generators $(\tilde{x}, \tilde{y}, \tilde{e})$ subject to the relations $[\tilde{e}, \tilde{x}]=n \tilde{x},[\tilde{e}, \tilde{y}]=-n \tilde{y}, \tilde{x} \tilde{y}=u(\tilde{e}), \tilde{y} \tilde{x}=u(\tilde{e}+n)$.

Remark 4.1.2. 1) The algebras $S(\mathbb{C}, f, n)$ were introduced and intensively studied by Smith [1990], who called them "algebras similar to $U\left(\mathfrak{s l}_{2}\right)$ ", where $U\left(\mathfrak{s l}_{2}\right)$ is the enveloping algebra of $\mathfrak{s l}_{2}$. In fact they share many interesting properties with $U\left(\mathfrak{s l}_{2}\right)$, in particular they have a very rich representation theory.

2) One can prove, as in [Smith 1990], that if the degree of $f$ is one and $n \neq 0$, and if the leading coefficient is invertible in $\boldsymbol{A}$, then $S(\boldsymbol{A}, f, n)$ is isomorphic to the enveloping algebra $U\left(\mathfrak{s l}_{2}(\boldsymbol{A})\right)$. 
Let $\mathscr{R}$ be a ring and let $\sigma \in \operatorname{Aut}(\mathscr{R})$. Let us recall that a $\sigma$-derivation of $\mathscr{R}$ is an additive map $\delta: \mathscr{R} \rightarrow \mathscr{R}$ such that $\delta(s u)=s \delta(u)+\delta(s) \sigma(u)$. Given a $\sigma$-derivation $\delta$, the skew polynomial ring over $\mathscr{R}$ determined by $\sigma$ and $\delta$ is the ring $\mathscr{R}[t, \sigma, \delta]:=\langle\mathscr{R}, t\rangle /\{s t-t \sigma(s)-\delta(s) \mid s \in \mathscr{R}\}$, where $\langle\mathscr{R}, t\rangle$ stands for the ring freely generated by $\mathscr{R}$ and an element $t$ with the relations given by the ring structure on $\mathscr{R}$ (for details see [McConnell and Robson 1987, Section 1.2, p. 15; Goodearl and Warfield 2004, p. 34]).

Proposition 4.1.3. Let $\mathfrak{b}$ the 2-dimensional Lie algebra over $\boldsymbol{A}$, with basis $\{\varepsilon, \alpha\}$ and relation $[\varepsilon, \alpha]=n \alpha$. Let $\mathcal{U}(\mathfrak{b})$ be the enveloping algebra of $\mathfrak{b}$. Define an automorphism $\sigma$ of $\mathcal{U}(\mathfrak{b})$ by $\sigma(\alpha)=\alpha$ and $\sigma(\varepsilon)=\varepsilon-n$ and define also $a$-derivation $\delta$ of $\mathcal{U}(\mathfrak{b})$ by $\delta(\alpha)=f(\varepsilon)$ and $\delta(\varepsilon)=0$. Then $S(\boldsymbol{A}, f, n) \simeq \mathcal{U}(\mathfrak{b})[t, \sigma, \delta]$.

Proof. The proof is almost the same as the one given by Smith [1990, Proposition 1.2]. The isomorphism $S(\boldsymbol{A}, f, n) \simeq \mathcal{U}(\mathfrak{b})[t, \sigma, \delta]$ is given by $e \mapsto \varepsilon, x \mapsto \alpha$ and $y \mapsto t$.

Corollary 4.1.4. $S(\boldsymbol{A}, f, n)$ is a noetherian domain with A-basis

$$
\left\{y^{i} x^{j} e^{k} \mid i, j, k \in \mathbb{N}\right\}
$$

(or any similar family of ordered monomials obtained by permutation of the elements $(y, x, e))$.

Proof. (compare with [Smith 1990, proof of Corollary 1.3, p. 288]). We know from [McConnell and Robson 1987, Theorem 1.2.9], that as $\mathcal{U}(\mathfrak{b})$ is a noetherian domain, so is $S(\boldsymbol{A}, f, n) \simeq \mathcal{U}(\mathfrak{b})[t, \sigma, \delta]$. Since

$$
\begin{aligned}
& \mathcal{U}(\mathfrak{b})[t, \sigma, \delta]=U(\mathfrak{b}) \oplus \mathcal{U}(\mathfrak{b}) t \oplus \mathcal{U}(\mathfrak{b}) t^{2} \oplus \mathcal{U}(\mathfrak{b}) t^{3} \oplus \cdots \oplus \mathcal{U}(\mathfrak{b}) t^{\ell} \oplus \cdots \\
& =U(\mathfrak{b}) \oplus t^{u} u(\mathfrak{b}) \oplus t^{2} u(\mathfrak{b}) \oplus t^{3} u(\mathfrak{b}) \oplus \cdots \oplus t^{\ell} u(\mathfrak{b}) \oplus \cdots
\end{aligned}
$$

(direct sums of $\boldsymbol{A}$-modules) and since the Poincaré-Birkhoff-Witt theorem is still true for enveloping algebras of Lie algebras which are free over rings (see [Bourbaki 1971]), the ordered monomials in $(y, x, e)$ beginning or ending with $y$ form a basis of the algebra $S(\boldsymbol{A}, f, n)$. To obtain the basis $\left\{e^{i} y^{j} x^{k}\right\}$ or $\left\{x^{k} y^{j} e^{i}\right\}$ it suffices to replace the algebra $\mathfrak{b}$ by the algebra $\mathfrak{b}_{-}$which is generated by $e$ and $y$.

Remark 4.1.5. The adjoint action of $e(u \mapsto[e, u])$ on $S(\boldsymbol{A}, f, n)$ is semisimple and gives a decomposition of $S(\boldsymbol{A}, f, n)$ into weight spaces:

$$
S(\boldsymbol{A}, f, n)=\bigoplus_{\nu \in \mathbb{Z}} S(\boldsymbol{A}, f, n)^{\nu},
$$

where $S(\boldsymbol{A}, f, n)^{v}=\{u \in S(\boldsymbol{A}, f, n) \mid[e, u]=v n u\}$. As $\left[e, x^{j} y^{i} e^{k}\right]=n(j-i) y^{i} x^{j} e^{k}$, we obtain, using Corollary 4.1.4, that the ordered monomials of the form $x^{i} y^{i} e^{k}$ form an $\boldsymbol{A}$-basis for $S(\boldsymbol{A}, f, n)^{0}$. Moreover as $y x=x y+f(e)$, it is easy to see 
that $S(\boldsymbol{A}, f, n)^{0}=\boldsymbol{A}[x y, e]=\boldsymbol{A}[y x, e]$, where $\boldsymbol{A}[x y, e]$ (resp. $\boldsymbol{A}[y x, e]$ ) denotes the $A$-subalgebra generated by $x y$ (resp. $y x$ ) and $e$.

The proof of the following lemma is straightforward.

Lemma 4.1.6. Let $n \in \mathbb{N}^{*}$ and let $f \in \boldsymbol{A}[t]$. There exists an element $u \in \boldsymbol{A}[t]$, which is unique up to addition of an element of $\boldsymbol{A}$, such that

$$
f(t)=u(t+n)-u(t)
$$

Proposition 4.1.7 (compare with [Smith 1990, Proposition 1.5]). Let $u$ be as in the preceding lemma. Define

$$
\Omega_{1}=x y-u(e) .
$$

Then the center of $S(\boldsymbol{A}, f, n)$ is $\boldsymbol{A}\left[\Omega_{1}\right]$ which is isomorphic to the polynomial algebra $\boldsymbol{A}[t]$.

Proof. Let us now prove that $\Omega_{1}$ is central. Obviously $\Omega_{1}$ commutes with $e$.

From the defining relations of $S(\boldsymbol{A}, f, n)$ we have $e x=x(e+n)$ and therefore, for any $k \in \mathbb{N}, e^{k} x=x(e+n)^{k}$.

This implies of course that for any polynomial $P \in A[t]$ we have

$$
P(e) x=x P(e+n) \quad \text { or } \quad P(e-n) x=x P(e) .
$$

Similarly one proves that

$$
P(e) y=y P(e-n) \quad \text { or } \quad P(e+n) y=y P(e) .
$$

Let us show that $\Omega_{1}$ commutes with $x$. Using Lemma 4.1.6 and (4-1-2) we obtain

$$
\begin{aligned}
x \Omega_{1} & =x(x y-u(e))=x^{2} y-x u(e)=x(y x-f(e))-x u(e) \\
& =x(y x-u(e+n)+u(e))-x u(e)=x y x-x u(e+n)=x y x-u(e) x \\
& =\Omega_{1} x .
\end{aligned}
$$

A similar calculation using (4-1-3) shows that $\Omega_{1}$ commutes also with $y$. Hence $\Omega_{1}$ belongs to the center of $S(\boldsymbol{A}, f, n)$.

Let now $z$ be a central element of $S(A, f, n)$. Then $z \in S(A, f, n)^{0}$. We have $S(\boldsymbol{A}, f, n)^{0}=\boldsymbol{A}[x y, e]=\boldsymbol{A}\left[\Omega_{1}, e\right]$, and hence $z$ can be written as follows:

$$
z=\sum c_{i}(e) \Omega_{1}^{i} \quad \text { (finite sum) }
$$

where $c_{i}(e) \in A[e]$. 
We have

$$
\begin{aligned}
0=[z, x] & =\left[\sum c_{i}(e) \Omega_{1}^{i}, x\right]=\sum\left[c_{i}(e), x\right] \Omega_{1}^{i} \\
& =\sum\left(c_{i}(e) x-x c_{i}(e)\right) \Omega_{1}^{i} \\
& =\sum x\left(c_{i}(e+n)-c_{i}(e)\right) \Omega_{1}^{i} \quad(\text { using (4-1-2)) } \\
& =x \sum\left(c_{i}(e+n)-c_{i}(e)\right) \Omega_{1}^{i} .
\end{aligned}
$$

As the algebra $S(\boldsymbol{A}, f, n)$ has no zero divisors we get

$$
\sum\left(c_{i}(e+n)-c_{i}(e)\right) \Omega_{1}^{i}=0 .
$$

As $\Omega_{1}=x y-u(e)$, we have $\Omega_{1}^{i}=x^{i} y^{i}$ modulo monomials of the form $e^{k} x^{p} y^{p}$ with $p<i$. Then from Corollary 4.1.4 above we obtain $c_{i}(e+n)-c_{i}(e)=0$ for all $i$. As the elements $e^{k}$ are free over $\boldsymbol{A}$ (Corollary 4.1.4) we obtain from Lemma 4.1.6 that $c_{i} \in \boldsymbol{A}$, for all $i$.

Remark 4.1.8. Conversely, let us start with $u \in \boldsymbol{A}[t]$. Define $f \in \boldsymbol{A}[t]$ by $f(t)=$ $u(t+n)-u(t)$. From the definitions we have

$$
U(\boldsymbol{A}, u, n)=S(\boldsymbol{A}, f, n) /(x y-u(e))=S(\boldsymbol{A}, f, n) /\left(\Omega_{1}\right),
$$

where $(x y-u(e))=\left(\Omega_{1}\right)$ is the ideal generated by $x y-u(e)=\Omega_{1}$. Again, as for $S(\boldsymbol{A}, f, n)$, the adjoint action of $\tilde{e}$ gives a decomposition of $U(\boldsymbol{A}, u, n)$ into weight spaces:

$$
U(\boldsymbol{A}, u, n)=\bigoplus_{\nu \in \mathbb{Z}} U(\boldsymbol{A}, u, n)^{v},
$$

where $U(\boldsymbol{A}, u, n)^{v}=\{\tilde{v} \in U(\boldsymbol{A}, u, n) \mid[\tilde{e}, \tilde{v}]=v n \tilde{v}\}$.

Proposition 4.1.9. Let $u \in A[t]$ and $s \in \mathbb{N}$. The A-linear mappings

$$
\varphi, \psi: \boldsymbol{A}[t] \rightarrow U(\boldsymbol{A}, u, n)
$$

given by

$$
\varphi(P)=\tilde{x}^{s} P(\tilde{e}), \quad \psi(P)=\tilde{y}^{s} P(\tilde{e})
$$

are injective (in particular the subalgebra $A[\tilde{e}] \subset U(\boldsymbol{A}, u, n)$ generated by $\tilde{e}$ is a polynomial algebra).

Proof. Define $f(t)=u(t+n)-u(t)$. Every element of $S(A, f, n)$ can be written uniquely in the form

$$
\sum a_{k, \ell, m} e^{k} x^{\ell} y^{m} \quad\left(a_{k, \ell, m} \in \boldsymbol{A}\right)
$$


(Corollary 4.1.4). Therefore, from Remark 4.1.8, every element in $U(\boldsymbol{A}, u, n)$ can be written in the form

$$
\sum a_{k, \ell, m} \tilde{e}^{k} \tilde{x}^{\ell} \tilde{y}^{m} \quad\left(a_{k, \ell, m} \in \boldsymbol{A}\right) .
$$

Let $P(t)=\sum_{i=0}^{p} a_{i} t^{i}\left(a_{i} \in \boldsymbol{A}\right)$ be a polynomial such that $\tilde{x}^{s} P(\tilde{e})=0$ (i.e., $\left.P \in \operatorname{ker} \varphi\right)$. As $U(\boldsymbol{A}, u, n)=S(\boldsymbol{A}, f, n) /\left(\Omega_{1}\right)$, we see that there exists $\alpha \in S(\boldsymbol{A}, f, n)$ such that

$$
x^{s} \sum_{i=0}^{p} a_{i} e^{i}=\alpha \Omega_{1}=\alpha(x y-u(e)) .
$$

If $\alpha=\sum a_{k, \ell, m} e^{k} x^{\ell} y^{m}$, using the fact that $\Omega_{1}=x y-u(e)$ is central and relation (4-1-2) we get

$$
\begin{aligned}
x^{s} \sum_{i=0}^{p} a_{i} e^{i} & =\left(\sum_{k, \ell, m} a_{k, \ell, m} e^{k} x^{\ell} y^{m}\right)(x y-u(e))=\sum_{k, \ell, m} a_{k, \ell, m} e^{k} x^{\ell}(x y-u(e)) y^{m} \\
& =\sum_{k, \ell, m} a_{k, \ell, m} e^{k} x^{\ell+1} y^{m+1}-\sum_{k, \ell, m} a_{k, \ell, m} e^{k} x^{\ell} u(e) y^{m} \\
& =\sum_{k, \ell, m} a_{k, \ell, m} e^{k} x^{\ell+1} y^{m+1}-\sum_{k, \ell, m} a_{k, \ell, m} e^{k} u(e-\ell n) x^{\ell} y^{m} .
\end{aligned}
$$

Suppose now that $\alpha \neq 0$; then one can define

$$
\ell_{0}=\max \left\{\ell \in \mathbb{N} \mid \exists k, m, a_{k, \ell, m} \neq 0\right\} .
$$

Let $k_{0}, m_{0}$ be such that $a_{k_{0}, \ell_{0}, m_{0}} \neq 0$. From (4-1-5) we get

$$
x^{s} \sum_{i=0}^{p} a_{i} e^{i}+\sum_{k, \ell, m} a_{k, \ell, m} e^{k} u(e-\ell n) x^{\ell} y^{m}=\sum_{k, \ell, m} a_{k, \ell, m} e^{k} x^{\ell+1} y^{m+1} .
$$

Using again (4-1-2) we obtain

$$
\sum_{i=0}^{p} a_{i}(e-n s)^{i} x^{s}+\sum_{k, \ell, m} a_{k, \ell, m} e^{k} u(e-\ell n) x^{\ell} y^{m}=\sum_{k, \ell, m} a_{k, \ell, m} e^{k} x^{\ell+1} y^{m+1} .
$$

The left side of this equality does not contain the monomial $e^{k_{0}} x^{\ell_{0}+1} y^{m_{0}+1}$, but the right side does. As the elements $e^{k} x^{\ell} y^{m}$ are a basis over $\boldsymbol{A}$ (Corollary 4.1.4), we obtain a contradiction. Therefore $\alpha=0$, and hence $x^{s} \sum_{i=0}^{p} a_{i} e^{i}$ vanishes. Again from Corollary 4.1.4, we obtain that $a_{i}=0$ for all $i$. This proves that $\operatorname{ker} \varphi=\{0\}$. The proof for $\psi$ is similar. 
Corollary 4.1.10. Every element $\tilde{u}$ in $U(\boldsymbol{A}, u, n)$ can be written uniquely in the form

$$
\tilde{u}=\sum_{\ell>0, k \geq 0} \alpha_{k, \ell} \tilde{y}^{\ell} \tilde{e}^{k}+\sum_{m \geq 0, r \geq 0} \beta_{m, r} \tilde{x}^{m} \tilde{e}^{r}
$$

with $\alpha_{k, \ell}, \beta_{m, r} \in \boldsymbol{A}$.

Proof. We have already noticed that any element in $U(\boldsymbol{A}, u, n)$ can be written (in a non unique way) as a linear combination, with coefficients in $A$, of the elements $\tilde{x}^{i} \tilde{y}^{j} \tilde{e}^{k}$.

Suppose that $i \geq j$. Then we have $\tilde{x}^{i} \tilde{y}^{j} \tilde{e}^{k}=\tilde{x}^{i-j} \tilde{x}^{j} \tilde{y}^{j} \tilde{e}^{k}$. As $\tilde{x} \tilde{y}=u(\tilde{e})$, we see that $\tilde{x}^{j} \tilde{y}^{j}=Q_{j}(\tilde{e})$, where $Q_{j}$ is a polynomial with coefficients in $\boldsymbol{A}$. Therefore $\tilde{x}^{i} \tilde{y}^{j} \tilde{e}^{k}=\sum_{\ell} \gamma_{\ell} \tilde{x}^{i-j} \tilde{e}^{\ell}$, with $\gamma_{\ell} \in \boldsymbol{A}$. Similarly one can prove that if $i<j$, we have $\tilde{x}^{i} \tilde{y}^{j} \tilde{e}^{k}=\sum_{\ell} \delta_{\ell} \tilde{y}^{j-i} \tilde{e}^{\ell}$, with $\delta_{\ell} \in \boldsymbol{A}$. This shows that any element $\tilde{u}$ in $U(\boldsymbol{A}, u, n)$ can be written in the expected form.

Suppose now that

$$
\sum_{\ell>0, k \geq 0} \alpha_{k, \ell} \tilde{y}^{\ell} \tilde{e}^{k}+\sum_{m \geq 0, r \geq 0} \beta_{m, r} \tilde{x}^{m} \tilde{e}^{r}=0 .
$$

Then, as $\tilde{y}^{\ell} \tilde{\boldsymbol{e}}^{k} \in U(\boldsymbol{A}, u, n)^{-\ell}$ and $\tilde{x}^{m} \tilde{\boldsymbol{e}}^{r} \in U(\boldsymbol{A}, u, n)^{m}$, we deduce from (4-1-4) that

$$
\sum_{k} \alpha_{k, \ell} \tilde{y}^{\ell} \tilde{e}^{k}=0 \quad \text { for all } \ell>0, \quad \sum_{r} \beta_{m, r} \tilde{x}^{m} \tilde{e}^{r}=0 \quad \text { for all } m \geq 0 .
$$

Then from Proposition 4.1.9, we deduce that $\alpha_{k, \ell}=0$ and $\beta_{m, r}=0$.

4.2. Generators and relations for $\boldsymbol{D}(\boldsymbol{V})^{\boldsymbol{G}^{\prime}}$. Let $\mathscr{L}(\mathscr{T})[t]$ be the polynomials in one variable with coefficients in $\mathscr{E}(\mathscr{T})$. From the commutation rules $[E, X]=d_{0} X$ and $[E, Y]=-d_{0} Y$, we easily deduce that for $P \in \mathscr{L}(\mathscr{T})[t]$ we have

$$
Y P(E)=P\left(E+d_{0}\right) Y, \quad X P(E)=P\left(E-d_{0}\right) X .
$$

From Theorem 3.2.6 above, we know that any element in $D(V)^{G}$ can be written uniquely as a polynomial in $E$ with coefficients in $\mathscr{L}(\mathscr{T})$. As $X Y$ and $Y X$ belong to $D(V)^{G}$, there exist therefore two uniquely determined polynomials $u_{X Y}$ and $u_{Y X}$ in $\mathscr{L}(\mathscr{T})[t]$ such that $X Y=u_{X Y}(E)$ and $Y X=u_{Y X}(E)$. From (4-2-1) we obtain that

$$
Y X Y=u_{Y X}(E) Y=Y u_{X Y}(E)=u_{X Y}\left(E+d_{0}\right) Y
$$

and therefore

$$
u_{Y X}(E)=u_{X Y}\left(E+d_{0}\right) .
$$

As the polynomial $u_{X Y}$ will play an important role in Theorem 4.2.2 below, let us emphasize the connection between $u_{X Y}$ and the Bernstein-Sato polynomial $b_{Y}$. 
Remark first that $b_{Y}=b_{X Y}$. We know from Corollary 3.2.8 that

$$
\begin{aligned}
h(X Y)(-\lambda+\rho) & =b_{X Y}(\lambda)=b_{Y}(\lambda) \\
& =\sum_{i=0}^{p} \alpha_{i}(-\lambda+\rho)\left(a_{0} d_{0}+a_{1} d_{1}+\cdots+a_{r} d_{r}\right)^{i} \\
& =\sum_{i=0}^{p} \alpha_{i}(-\lambda+\rho)(h(E)(-\lambda+\rho))^{i}
\end{aligned}
$$

with uniquely defined polynomials $\alpha_{i} \in \mathbb{C}[A]^{W_{0}, \tau}$. Therefore we obtain

Proposition 4.2.1. Keeping the notation above, we have

$$
u_{X Y}(t)=\sum_{i=0}^{p} h^{-1}\left(\alpha_{i}\right) t^{i} .
$$

Theorem 4.2.2. Let $f_{X Y}(t)=u_{X Y}\left(t+d_{0}\right)-u_{X Y}(t)$. The mapping

$$
\tilde{x} \mapsto X, \quad \tilde{y} \mapsto Y, \quad \tilde{e} \mapsto E
$$

extends uniquely to an isomorphism of $\mathscr{L}(\mathscr{T})$-algebras between $U\left(\mathscr{L}(\mathscr{T}), u_{X Y}, d_{0}\right)$ (which is isomorphic to $S\left(\mathscr{L}(\mathscr{T}), f_{X Y}, d_{0}\right) /\left(\Omega_{1}\right)$ ) and $D(V)^{G^{\prime}}=\mathscr{T}_{0}[X, Y]$.

Proof. As $[E, X]=d_{0} X,[E, Y]=-d_{0} Y, X Y=u_{X Y}(E)$ and $Y X=u_{X Y}\left(E+d_{0}\right)$ (see (4-2-2)), and as from Theorem 3.2.6 the algebra $D(V)^{G^{\prime}}=\mathscr{T}_{0}[X, Y]$ is generated over $\mathscr{L}(\mathscr{T})$ by $X, Y, E$, we know (universal property) that the mapping

$$
\tilde{x} \mapsto X, \quad \tilde{y} \mapsto Y, \quad \tilde{e} \mapsto E
$$

extends uniquely to a surjective morphism of $\mathscr{L}(\mathscr{T})$-algebras:

$$
\varphi: U\left(\mathscr{E}(\mathscr{T}), u_{X Y}, d_{0}\right) \rightarrow D(V)^{G^{\prime}} .
$$

From Corollary 4.1 .10 any element $\tilde{u}$ in $U\left(\mathscr{L}(\mathscr{T}), u_{X Y}, d_{0}\right)$ can be written uniquely in the form

$$
\tilde{u}=\sum_{\ell>0, k \geq 0} \alpha_{k, \ell} \tilde{y}^{\ell} \tilde{e}^{k}+\sum_{m \geq 0, r \geq 0} \beta_{m, r} \tilde{x}^{m} \tilde{e}^{r}
$$

with $\alpha_{k, \ell}, \beta_{m, r} \in \mathscr{L}(\mathscr{T})$. Suppose now that $\tilde{u} \in \operatorname{ker}(\varphi)$, then

$$
\varphi(\tilde{u})=\sum_{\ell>0, k \geq 0} \alpha_{k, \ell} Y^{\ell} E^{k}+\sum_{m \geq 0, r \geq 0} \beta_{m, r} X^{m} E^{r}=0,
$$

with $\alpha_{k, \ell}, \beta_{m, r} \in \mathscr{L}(\mathscr{T})$. Then Corollary 3.2.7 implies that $\alpha_{k, \ell}=\beta_{m, r}=0$. Hence $\varphi$ is an isomorphism. 


\section{Radial components}

5.1. Radial components and Bernstein-Sato polynomials. Remember that for $\tilde{\boldsymbol{a}}=\left(a_{1}, a_{2}, \ldots, a_{r}\right) \in \mathbb{N}^{r}$ we have defined $V_{\tilde{\boldsymbol{a}}}=V_{\left(0, a_{1}, \ldots, a_{r}\right)}$. Remember also that for $\boldsymbol{a}=\left(a_{0}, a_{1}, \ldots, a_{r}\right)$ we have $V_{\boldsymbol{a}}=\Delta^{a_{0}} V_{\tilde{\boldsymbol{a}}}$. We know from Proposition 2.2.8 that the spaces $U_{\tilde{\boldsymbol{a}}}=\bigoplus_{a_{0} \in \mathbb{N}} \Delta_{0}^{a_{0}} V_{\tilde{\boldsymbol{a}}}$ are the $G^{\prime}$-isotypic components of $\mathbb{C}[V]$ and that the spaces $W_{\tilde{\boldsymbol{a}}}=\bigoplus_{a_{0} \in \mathbb{Z}} \Delta_{0}^{a_{0}} V_{\tilde{\boldsymbol{a}}}$ are the $G^{\prime}$-isotypic components of $\mathbb{C}[\mathbb{O}]$. Therefore the algebra $D(V)^{G^{\prime}}=\mathscr{T}_{0}[X, Y]$ stabilizes each space $U_{\tilde{a}}$ and the algebra $D(\mathcal{O})^{G^{\prime}}=\mathscr{T}_{0}\left[X, X^{-1}\right]=\mathscr{T}$ stabilizes each space $W_{\tilde{\boldsymbol{a}}}$.

Let us consider the restriction map

$$
\begin{aligned}
D(\mathcal{O})^{G^{\prime}} & \rightarrow \operatorname{End}\left(W_{\tilde{\boldsymbol{a}}}\right), \\
D & \mapsto r_{\tilde{\boldsymbol{a}}}(D)=D_{\mid W_{\tilde{\boldsymbol{a}}}} .
\end{aligned}
$$

Definition 5.1.1. Let $D \in D(\mathcal{O})^{G^{\prime}}=\mathscr{T}_{0}\left[X, X^{-1}\right]=\mathscr{T}$. The operator $r_{\tilde{\boldsymbol{a}}}(D)=D_{\left.\right|_{\tilde{a}}}$ is called the radial component of $D$ with respect to $\tilde{\boldsymbol{a}}$.

Example 5.1.2. Consider the case where $\tilde{\boldsymbol{a}}=0$. Then $W_{\tilde{\boldsymbol{a}}}=\mathbb{C}\left[\Delta_{0}, \Delta_{0}^{-1}\right]$, and $r_{0}(D)$ is the endomorphism of $\mathbb{C}\left[t, t^{-1}\right]$ defined by $D\left(\varphi \circ \Delta_{0}\right)=r_{0}(D)(\varphi) \circ \Delta_{0}$. The operator $r_{0}(D)$ is the usual radial component of $D$ (we will see below that it is a differential operator).

Notice now that the space $W_{\tilde{\boldsymbol{a}}}=\bigoplus_{a_{0} \in \mathbb{Z}} \Delta_{0}^{a_{0}} V_{\tilde{\boldsymbol{a}}}$ can be viewed as the space of Laurent polynomials in $\Delta_{0}$, with coefficients in $V_{\tilde{a}}$, in other words any $P \in W_{\tilde{a}}$ can be written uniquely under the form

$$
P=\sum \Delta_{0}^{p} \gamma_{p}
$$

with $\gamma_{p} \in V_{\tilde{\boldsymbol{a}}}$. This can also be written as $P=\varphi \circ\left(\Delta_{0}\right)$, with $\varphi(t)=\sum t^{p} \gamma_{p}$ in $V_{\tilde{a}}\left[t, t^{-1}\right]$ (this being precisely the set of linear combinations $\sum t^{p} \gamma_{p}$, with $\left.\gamma_{p} \in V_{\tilde{\boldsymbol{a}}}\right)$.

There is a natural action of $D\left(\mathbb{C}^{*}\right)=\mathbb{C}\left[t, t^{-1}, t d / d t\right]$ on $V_{\tilde{a}}\left[t, t^{-1}\right]$ given by $(d / d t) t^{p} \gamma_{p}=p t^{p-1} \gamma_{p}$.

Proposition 5.1.3. Let $D \in \mathscr{T}_{n}$ a homogeneous element of degree $n$. Let $b_{D}$ be its Bernstein-Sato polynomial. Let $\varphi \in V_{\tilde{a}}\left[t, t^{-1}\right]$. Then

$$
D\left(\varphi \circ \Delta_{0}\right)=\left(t^{n} b_{D}\left(t d / d t, a_{1}, \ldots, a_{r}\right) \varphi\right) \circ \Delta_{0} ;
$$

in other words, $r_{\tilde{\boldsymbol{a}}}(D)=t^{n} b_{D}\left(t d / d t, a_{1}, \ldots, a_{r}\right)$.

Proof. It is enough to show that the two operators coincide on elements of the form $\Delta_{0}^{p} \gamma_{p}$, with $\gamma_{p} \in V_{\tilde{\boldsymbol{a}}}$. Then $\varphi=t^{p} \gamma_{p}$. Let us write

$$
b_{D}(\boldsymbol{a})=\sum_{k} c_{k}\left(a_{1}, \ldots, a_{r}\right) a_{0}^{k} .
$$


We have

$$
\begin{aligned}
\left(t^{n} b_{D}\left(t \frac{d}{d t}, a_{1}, \ldots, a_{r}\right) \varphi\right) \circ \Delta_{0} & =t^{n}\left(\sum_{k} c_{k}\left(a_{1}, \ldots, a_{r}\right)\left(t \frac{d}{d t}\right)^{k} \varphi\right) \circ \Delta_{0} \\
& =t^{n}\left(\sum_{k} c_{k}\left(a_{1}, \ldots, a_{r}\right) p^{k} t^{p} \gamma_{p}\right) \circ \Delta_{0} \\
& =\left(t^{n} b_{D}\left(p, a_{1}, \ldots, a_{r}\right) t^{p} \gamma_{p}\right) \circ \Delta_{0} \\
& =b_{D}\left(p, a_{1}, \ldots, a_{r}\right) \Delta_{0}^{p+n} \gamma_{p} \\
& =D\left(\Delta_{0}^{p} \gamma_{p}\right) .
\end{aligned}
$$

Corollary 5.1.4. If $(G, V)$ is a $P V$ of commutative parabolic type of rank $r+1$, then the radial component of $Y$ is given by

$$
r_{\tilde{\boldsymbol{a}}}(Y)=c t^{-1} \prod_{j=0}^{r}\left(t \frac{d}{d t}+a_{1}+\cdots+a_{j}+j \frac{d}{2}\right) .
$$

Proof. This is just a consequence of the formula for $b_{Y}$ given in Example 3.1.2.

Example 5.1.5. Consider case 1) in Example 2.3.3: then $G=(\operatorname{SL}(n) \times \operatorname{SL}(n)) \times \mathbb{C}^{*}$ acting on $x \in V=M_{n}(\mathbb{C})$ by $\left(g_{1}, g_{2}, t\right) \cdot x=\operatorname{tg}_{1} x g_{2}^{-1}$. Then $\Delta_{0}=X=\operatorname{det} x$ and

$$
Y=\Delta_{0}^{*}(\partial)=\operatorname{det}\left(\frac{\partial}{\partial x_{i j}}\right)
$$

where $x_{i j}$ are the coefficients of the matrix $X$. As in this case $d / 2=1$ (see [Muller et al. 1986, Table 2, p. 122]), we have

$$
b_{Y}\left(a_{0}, a_{1}, \ldots, a_{n-1}\right)=\prod_{j=0}^{n-1}\left(a_{0}+a_{1}+\cdots+a_{j}+j\right) .
$$

Hence the radial component $r_{0}(Y)$ defined by $\operatorname{det}\left(\frac{\partial}{\partial x_{i j}}\right)(\varphi \circ \operatorname{det})=\left(r_{0}(Y) \varphi\right) \circ \operatorname{det}$ is given by

$$
r_{0}(Y)=t^{-1} \prod_{j=0}^{n-1}\left(t \frac{d}{d t}+j\right) .
$$

This radial component has already been calculated by Raïs [1972, p. 22], by other methods. He obtained that $r_{0}(Y)=\left[\prod_{j=2}^{n}(t d / d t+j)\right] d / d t$. A simple calculation shows that the two operators are the same.

\subsection{Algebras of radial components.}

Definition 5.2.1. The radial component algebra $R_{\tilde{a}}$ is the image of $D(V)^{G^{\prime}}=$ $\mathscr{T}_{0}[X, Y]$ under the map $D \mapsto r_{\tilde{\boldsymbol{a}}}(D)$. 
Remember from Proposition 3.2.3 that the elements $D$ in $\mathscr{L}(\mathscr{T})$ are characterized by the fact that the corresponding Bernstein-Sato polynomial $b_{D}$ does not depend on the $a_{0}$ variable. Therefore such a $D$ acts by the scalar $b_{D}(0, \tilde{\boldsymbol{a}})$ on $W_{\tilde{\boldsymbol{a}}}$; that is, $r_{\tilde{\boldsymbol{a}}}(D)=b_{D}(0, \tilde{\boldsymbol{a}}) I d_{W_{\tilde{a}}}$.

Let us consider the polynomial $u_{X Y} \in \mathscr{L}(\mathscr{T})[t]$ introduced in Section 4.2. If $u_{X Y}=\sum_{i} c_{i} t^{i}$, with $c_{i} \in \mathscr{L}(\mathscr{T})$, we define

$$
r_{\tilde{\boldsymbol{a}}}\left(u_{X Y}\right)=\sum_{i} r_{\tilde{\boldsymbol{a}}}\left(c_{i}\right) t^{i} \in \mathbb{C}[t] .
$$

Lemma 5.2.2. Let $\boldsymbol{a}=\left(a_{0}, a_{1}, \ldots, a_{r}\right) \in \mathbb{N}^{r+1}$. Suppose that $a_{0}>0$. Then the map $P \mapsto Y P$ from $V_{\boldsymbol{a}}$ to $V_{\boldsymbol{a}-1}$ is a $G^{\prime}$-equivariant isomorphism.

Sketch of proof. It is enough to prove that this map is not 0 . As $\Delta_{0}^{* a_{0}} \cdots \Delta_{r}^{* a_{r}}$ is the lowest weight vector of $V_{\boldsymbol{a}}^{*} \subset \mathbb{C}\left[V^{*}\right]$ we have $\Delta_{0}^{*}(\partial)^{a_{0}} \cdots \Delta_{r}^{*}(\partial)^{a_{r}} \Delta_{0}^{a_{0}} \cdots \Delta_{r}^{a_{r}}(0) \neq 0$. Hence $\Delta_{0}^{*}(\partial) \Delta_{0}^{a_{0}} \cdots \Delta_{r}^{a_{r}} \neq 0$.

Theorem 5.2.3. The radial component algebra $R_{\tilde{a}}$ is isomorphic, as an associative algebra over $\mathbb{C}$, to the algebra $U\left(\mathbb{C}, r_{\tilde{a}}\left(u_{X Y}\right), d_{0}\right)$ introduced in Definition 4.1.1.

Proof. The algebra $R_{\tilde{a}}$ is generated over $\mathbb{C}$ by the elements $r_{\tilde{a}}(E), r_{\tilde{a}}(X), r_{\tilde{a}}(Y)$. The defining relations of $U\left(\mathbb{C}, r_{\tilde{\boldsymbol{a}}}\left(u_{X Y}\right), d_{0}\right)$ are satisfied:

$$
\begin{aligned}
{\left[r_{\tilde{\boldsymbol{a}}}(E), r_{\tilde{\boldsymbol{a}}}(X)\right] } & =r_{\tilde{\boldsymbol{a}}}([E, X])=d_{0} r_{\tilde{\boldsymbol{a}}}(X), \\
{\left[r_{\tilde{\boldsymbol{a}}}(E), r_{\tilde{\boldsymbol{a}}}(Y)\right] } & =r_{\tilde{\boldsymbol{a}}}([E, Y])=-d_{0} r_{\tilde{\boldsymbol{a}}}(Y), \\
r_{\tilde{\boldsymbol{a}}}(X) r_{\tilde{\boldsymbol{a}}}(Y) & =r_{\tilde{\boldsymbol{a}}}(X Y)=r_{\tilde{\boldsymbol{a}}}\left(u_{X Y}\right)\left(r_{\tilde{\boldsymbol{a}}}(E)\right), \\
r_{\tilde{\boldsymbol{a}}}(Y) r_{\tilde{\boldsymbol{a}}}(X) & =r_{\tilde{\boldsymbol{a}}}(Y X)=r_{\tilde{\boldsymbol{a}}}\left(u_{X Y}\right)\left(r_{\tilde{\boldsymbol{a}}}(E)+d_{0}\right) .
\end{aligned}
$$

Therefore the mapping

$$
\tilde{x} \mapsto r_{\tilde{\boldsymbol{a}}}(X), \quad \tilde{y} \mapsto r_{\tilde{\boldsymbol{a}}}(Y), \quad \tilde{e} \mapsto r_{\tilde{\boldsymbol{a}}}(E)
$$

extends uniquely to a surjective morphism of $\mathbb{C}$-algebras

$$
\varphi_{\tilde{\boldsymbol{a}}}: U\left(\mathbb{C}, r_{\tilde{\boldsymbol{a}}}\left(u_{X Y}\right), d_{0}\right) \rightarrow R_{\tilde{\boldsymbol{a}}}
$$

From Corollary 4.1 .10 any element $\tilde{u}$ in $U\left(\mathbb{C}, r_{\tilde{\boldsymbol{a}}}\left(u_{X Y}\right), d_{0}\right)$ can be written uniquely in the form

$$
\tilde{u}=\sum_{\ell>0, k \geq 0} \alpha_{k, \ell} \tilde{y}^{\ell} \tilde{e}^{k}+\sum_{m \geq 0, s \geq 0} \beta_{m, s} \tilde{x}^{m} \tilde{e}^{s}
$$

with $\alpha_{k, \ell}, \beta_{m, s} \in \mathbb{C}$. Suppose now that $\tilde{u} \in \operatorname{ker}\left(\varphi_{\tilde{a}}\right)$, then

$$
\varphi_{\tilde{\boldsymbol{a}}}(\tilde{u})=\sum_{\ell>0, k \geq 0} \alpha_{k, \ell} r_{\tilde{\boldsymbol{a}}}(Y)^{\ell} r_{\tilde{\boldsymbol{a}}}(E)^{k}+\sum_{m \geq 0, s \geq 0} \beta_{m, s} r_{\tilde{\boldsymbol{a}}}(X)^{m} r_{\tilde{\boldsymbol{a}}}(E)^{s}=0 .
$$


Applying this operator to a function of the form $\Delta^{a_{0}} P$, with $P \in V_{\tilde{a}}$, we obtain

$$
\sum_{\ell>0} Y^{\ell}\left(\sum_{k \geq 0} \alpha_{k, \ell} E^{k} \Delta^{a_{0}} P\right)+\sum_{m \geq 0} X^{m}\left(\sum_{s \geq 0} \beta_{m, s} E^{s} \Delta^{a_{0}} P\right)=0 .
$$

As the operators $X$ and $Y$ have degree $d_{0}$ and $-d_{0}$, respectively, this implies that

$$
\begin{aligned}
& Y^{\ell}\left(\sum_{k \geq 0} \alpha_{k, \ell} E^{k} \Delta^{a_{0} P}\right)=0 \text { for all } \ell, \\
& X^{m}\left(\sum_{s \geq 0} \beta_{m, s} E^{s} \Delta^{a_{0}} P\right)=0 \text { for all } m \text {. }
\end{aligned}
$$

Therefore, by Lemma 5.2.2 we obtain $\sum_{k \geq 0} \alpha_{k, \ell} E^{k} \Delta^{a_{0}} P=0$ for all $\ell$ and all $a_{0}>\ell$, and $\sum_{s \geq 0} \beta_{m, s} E^{s} \Delta^{a_{0}} P=0$ for all $m, a_{0}$. As $E \Delta^{a_{0}} P=\left(a_{0} d_{0}+d(\tilde{\boldsymbol{a}})\right) \Delta^{a_{0}} P$, where $d(\tilde{\boldsymbol{a}})=a_{1} d_{1}+\cdots+a_{r} d_{r}$, we have $\sum_{k \geq 0} \alpha_{k, \ell}\left(a_{0} d_{0}+d(\tilde{\boldsymbol{a}})\right)^{k} \Delta^{a_{0}} P=0$ for all $\ell$ and all $a_{0}>\ell$, and $\sum_{s \geq 0} \beta_{m, s}\left(a_{0} d_{0}+d(\tilde{\boldsymbol{a}})\right)^{s} \Delta^{a_{0}} P=0$ for all $m, a_{0}$. Hence $\sum_{k \geq 0} \alpha_{k, \ell}\left(a_{0} d_{0}+d(\tilde{\boldsymbol{a}})\right)^{k}=0$ for all $\ell$ and $a_{0}>\ell$, and $\sum_{s \geq 0} \beta_{m, s}\left(a_{0} d_{0}+d(\tilde{\boldsymbol{a}})\right)^{s}=0$ for all $m, a_{0}$. This implies that $\alpha_{k, \ell}=0$ and $\beta_{m, s}=0$ for all $\ell, k, m, s$. Hence $\tilde{u}=0$ and $\varphi_{\tilde{a}}$ is injective.

Remark 5.2.4. For $\tilde{\boldsymbol{a}}=0$, the preceding result was first obtained by Levasseur [2009], by other methods.

Now define $J_{\tilde{\boldsymbol{a}}}=\operatorname{ker}\left(r_{\left.\tilde{\boldsymbol{a}}\right|_{D(V) G^{\prime}}}\right)$. This is a two-sided ideal of $D(V)^{G^{\prime}}=\mathscr{T}_{0}[X, Y]$. Remember from Proposition 3.1.6 that any $D \in D(V)^{G^{\prime}}$ can be written uniquely in the form

$$
D=\sum_{k \in \mathbb{N}^{*}} u_{k} Y^{k}+\sum_{n \in \mathbb{N}} v_{n} X^{n} \quad \text { (finite sums), }
$$

where $u_{k}, v_{n} \in \mathscr{T}_{0}=D(V)^{G}$.

Lemma 5.2.5. $J_{\tilde{\boldsymbol{a}}}=\left\{D=\sum_{k \in \mathbb{N}^{*}} u_{k} Y^{k}+\sum_{n \in \mathbb{N}} v_{n} X^{n} \mid u_{k}, v_{n} \in J_{\tilde{\boldsymbol{a}}} \cap \mathscr{T}_{0}\right\}$.

Proof. From Theorem 5.2.3 the algebra $R_{\tilde{a}}$ is isomorphic to $U\left(\mathbb{C}, r_{\tilde{\boldsymbol{a}}}\left(u_{X Y}\right), d_{0}\right)$. If $r_{\tilde{\boldsymbol{a}}}(D)=\sum_{k \in \mathbb{N}^{*}} r_{\tilde{\boldsymbol{a}}}\left(u_{k}\right) r_{\tilde{\boldsymbol{a}}}(Y)^{k}+\sum_{n \in \mathbb{N}} r_{\tilde{\boldsymbol{a}}}\left(v_{n}\right) r_{\tilde{\boldsymbol{a}}}(X)^{n}=0$, then from Corollary 4.1.10 we obtain that $r_{\tilde{\boldsymbol{a}}}\left(u_{k}\right)=0$ and $r_{\tilde{\boldsymbol{a}}}\left(v_{n}\right)=0$ for all $k$ and all $n$.

Let us now give a set of generators for the ideal $\operatorname{ker}\left(r_{\tilde{\boldsymbol{a}}}\right)$ in $D(V)^{G^{\prime}}=\mathscr{T}_{0}[X, Y]$. From Proposition 5.1.3 we obtain that $r_{\tilde{\boldsymbol{a}}}(E)=d_{0}(t d / d t)+d(\tilde{\boldsymbol{a}})$. Therefore $r_{\tilde{\boldsymbol{a}}}\left((E-d(\tilde{\boldsymbol{a}})) / d_{0}\right)=t d / d t$. Define $G_{i}^{\tilde{\boldsymbol{a}}}=R_{i}-b_{R_{i}}\left((E-d(\tilde{\boldsymbol{a}})) / d_{0}, \tilde{\boldsymbol{a}}\right)$, where the $R_{i}$ are the Capelli operators introduced in Section 2.2. Obviously $G_{i}^{\tilde{a}} \in D(V)^{G}=\mathscr{T}_{0}$. 
Using Proposition 5.1.3 again we obtain

$$
\begin{aligned}
r_{\tilde{\boldsymbol{a}}}\left(G_{i}^{\tilde{\boldsymbol{a}}}\right) & =r_{\tilde{\boldsymbol{a}}}\left(R_{i}-b_{R_{i}}\left(\frac{E-d(\tilde{\boldsymbol{a}})}{d_{0}}, \tilde{\boldsymbol{a}}\right)\right) \\
& =r_{\tilde{\boldsymbol{a}}}\left(R_{i}\right)-b_{R_{i}}\left(r_{\tilde{\boldsymbol{a}}}\left(\frac{E-d(\tilde{\boldsymbol{a}})}{d_{0}}\right), \tilde{\boldsymbol{a}}\right) \\
& =b_{R_{i}}\left(t \frac{d}{d t}, \tilde{\boldsymbol{a}}\right)-b_{R_{i}}\left(t \frac{d}{d t}, \tilde{\boldsymbol{a}}\right)=0 .
\end{aligned}
$$

Hence the elements $G_{i}^{\tilde{a}}$ belong to $J_{\tilde{\boldsymbol{a}}} \cap \mathscr{T}_{0}$.

Theorem 5.2.6. The elements $G_{i}^{\tilde{a}}$ are generators of $J_{\tilde{\boldsymbol{a}}}$ :

$$
J_{\tilde{\boldsymbol{a}}}=\operatorname{ker}\left(\left.r_{\tilde{\boldsymbol{a}}}\right|_{D(V)^{G^{\prime}}}\right)=\sum_{i=0}^{r} D(V)^{G^{\prime}} G_{i}^{\tilde{a}}=\sum_{i=0}^{r} G_{i}^{\tilde{a}} D(V)^{G^{\prime}} .
$$

Proof. From Lemma 5.2.5, it is now enough to prove that

$$
J_{\tilde{\boldsymbol{a}}} \cap \mathscr{T}_{0} \subset \sum_{i=0}^{r} D(V)^{G} G_{i}^{\tilde{a}}=\sum_{i=0}^{r} \mathscr{T}_{0} G_{i}^{\tilde{\boldsymbol{a}}} .
$$

Let $D \in J_{\tilde{a}} \cap \mathscr{T}_{0}$. As $\mathscr{T}_{0}=\mathbb{C}\left[R_{0}, \ldots, R_{r}\right]$ (Theorem 2.2.9), we have also $\mathscr{T}_{0}=\mathbb{C}\left[G_{0}^{\tilde{a}}, \ldots, G_{r}^{\tilde{a}}, E\right]$. Therefore $D=\sum Q_{i} E^{i}$, where $Q_{i} \in \mathbb{C}\left[G_{0}^{\tilde{a}}, \ldots, G_{r}^{\tilde{a}}\right]$. Hence $Q_{i} \in Q_{i}(0)+\sum_{i=0}^{r} D(V)^{G} G_{i}^{\tilde{a}}$. Then

$$
0=r_{\tilde{\boldsymbol{a}}}(D)=\sum_{i} Q_{i}(0) r_{\tilde{\boldsymbol{a}}}\left(E^{i}\right)=\sum_{i} Q_{i}(0)\left(d_{0}\left(t \frac{d}{d t}\right)+d(\tilde{\boldsymbol{a}})\right)^{i} .
$$

Therefore $Q_{i}(0)=0(i=0, \ldots, r)$. Hence $Q_{i} \in \sum_{i=0}^{r} D(V)^{G} G_{i}^{\tilde{a}}$, which yields $D \in \sum_{i=0}^{r} D(V)^{G} G_{i}^{\tilde{a}}$.

Remark 5.2.7. For $\tilde{\boldsymbol{a}}=0$, the result of the preceding theorem is due to [Levasseur 2009, Theorem 4.11(v)].

\subsection{Rational radial component algebras.}

Definition 5.3.1. The rational radial component algebra $R_{\tilde{a}}^{r}$ is the image of

$$
D(\mathcal{O})^{G^{\prime}}=\mathscr{T}_{0}\left[X, X^{-1}\right]=\mathscr{T}
$$

under the map $D \mapsto r_{\tilde{a}}(D)$.

In fact as shown in the following proposition the structure of the algebras $R_{\tilde{a}}^{r}$ is simpler than the structure of $R_{\tilde{a}}$, and the ideal $I_{\tilde{a}}=\operatorname{ker}\left(r_{\tilde{a}}\right) \subset \mathscr{T}$ has the same generators as $J_{\tilde{a}}$.

Proposition 5.3.2. 1) For all $\tilde{\boldsymbol{a}}$, the rational radial component algebra $R_{\tilde{a}}^{r}$ is isomorphic to $\mathbb{C}\left[t, t^{-1}, t d / d t\right]$. 
2) $I_{\tilde{\boldsymbol{a}}}=\operatorname{ker}\left(r_{\tilde{\boldsymbol{a}}}\right)=\sum_{i=0}^{r} \mathscr{T} G_{i}^{\tilde{\boldsymbol{a}}}=\sum_{i=0}^{r} G_{i}^{\tilde{\boldsymbol{a}}} \mathscr{T}$.

Proof. 1) We have $\mathscr{T}=\mathscr{T}_{0}\left[X, X^{-1}\right]$. And $\mathscr{T}_{0}=\mathscr{L}(\mathscr{T})[E]$, from Theorem 3.2.6. Therefore $\mathscr{T}=\mathscr{L}(\mathscr{T})\left[X, X^{-1}, E\right]$. On the other hand we have $r_{\tilde{a}}(\mathscr{L}(\mathscr{T}))=\mathbb{C}$, $r_{\tilde{\boldsymbol{a}}}(X)=t, r_{\tilde{\boldsymbol{a}}}\left(X^{-1}\right)=t^{-1}$ and $r_{\tilde{\boldsymbol{a}}}(E)=d_{0}(t d / d t)+d(\tilde{\boldsymbol{a}})$. Hence

$$
R_{\tilde{\boldsymbol{a}}}^{r}=r_{\tilde{\boldsymbol{a}}}(\mathcal{T})=\mathbb{C}\left[t, t^{-1}, d_{0}\left(t \frac{d}{d t}\right)+d(\tilde{\boldsymbol{a}})\right]=\mathbb{C}\left[t, t^{-1}, t \frac{d}{d t}\right] .
$$

2) Obviously $\sum_{i=0}^{r} \mathscr{T} G_{i}^{\tilde{a}} \subset I_{\tilde{\boldsymbol{a}}}$. As $I_{\tilde{\boldsymbol{a}}}$ is a two-sided ideal of $\mathscr{T}$, it is easily seen to be graded. If $D \in I_{\tilde{\boldsymbol{a}}} \cap \mathcal{T}_{p}$, then $X^{-p} D \in \mathscr{T}_{0} \cap I_{\tilde{\boldsymbol{a}}}=\mathscr{T}_{0} \cap J_{\tilde{\boldsymbol{a}}}=\sum_{i=0}^{r} \mathcal{T}_{0} G_{i}^{\tilde{\boldsymbol{a}}}$. Therefore $D \in \sum_{i=0}^{r} \mathscr{T} G_{i}^{\tilde{a}}$.

\section{Acknowledgements}

I would like to thank Thierry Levasseur for providing me with the manuscript of [Levasseur 2009]. I would also like to thank Sylvain Rubenthaler who provided me with a first proof of Proposition 4.1.9 which was important for my understanding.

\section{References}

[Benson and Ratcliff 1996] C. Benson and G. Ratcliff, "A classification of multiplicity free actions", J. Algebra 181:1 (1996), 152-186. MR 97c:14046 Zbl 0869.14021

[Benson and Ratcliff 2000] C. Benson and G. Ratcliff, "Rationality of the generalized binomial coefficients for a multiplicity free action”, J. Austral. Math. Soc. Ser. A 68:3 (2000), 387-410. MR 2001a:20071 Zbl 0964.20021

[Benson and Ratcliff 2004] C. Benson and G. Ratcliff, "On multiplicity free actions", pp. 221-304 in Representations of real and p-adic groups (Singapore, 2002-2003), edited by E.-C. Tan and C.-B. Zhu, Lect. Notes Ser. Inst. Math. Sci. Natl. Univ. Singap. 2, World Scientific, River Edge, NJ, 2004. MR 2005k:20107 Zbl 1061.22017

[Van den Bergh 1996] M. Van den Bergh, "Some rings of differential operators for $\mathrm{Sl}_{2}$-invariants are simple”, J. Pure Appl. Algebra 107:2-3 (1996), 309-335. MR 97c:16032 Zbl 0871.16014

[Bopp and Rubenthaler 1993] N. Bopp and H. Rubenthaler, "Fonction zêta associée à la série principale sphérique de certains espaces symétriques", Ann. Sci. École Norm. Sup. (4) 26:6 (1993), 701-745. MR 95f:11095 Zbl 0806.14039

[Bopp and Rubenthaler 2005] N. Bopp and H. Rubenthaler, "Local zeta functions attached to the minimal spherical series for a class of symmetric spaces", Mem. Amer. Math. Soc. 174:821 (2005). MR 2006c:11140 Zbl 1076.11059

[Bourbaki 1971] N. Bourbaki, Groupes et algèbres de Lie, chapitre I: Algèbres de Lie, 2nd ed., Actualités Scientifiques et Industrielles 1285, Hermann, Paris, 1971. MR 42 \#6159 Zbl 0213.04103

[Brion 1985] M. Brion, "Représentations exceptionnelles des groupes semi-simples", Ann. Sci. École Norm. Sup. (4) 18:2 (1985), 345-387. MR 87e:14043 Zbl 0588.22010

[Faraut and Korányi 1994] J. Faraut and A. Korányi, Analysis on symmetric cones, Clarendon/Oxford University Press, New York, 1994. MR 98g:17031 Zbl 0841.43002 
[Goodearl and Warfield 2004] K. R. Goodearl and R. B. Warfield, Jr., An introduction to noncommutative Noetherian rings, 2nd ed., London Mathematical Society Student Texts 61, Cambridge University Press, 2004. MR 2005b:16001 Zbl 1101.16001

[Heckman and Schlichtkrull 1994] G. Heckman and H. Schlichtkrull, Harmonic analysis and special functions on symmetric spaces, Perspectives in Mathematics 16, Academic Press, San Diego, CA, 1994. MR 96j:22019 Zbl 0836.43001

[Howe and Umeda 1991] R. Howe and T. Umeda, "The Capelli identity, the double commutant theorem, and multiplicity-free actions", Math. Ann. 290:3 (1991), 565-619. MR 92j:17004 Zbl 0733.20019

[Igusa 1981] J.-I. Igusa, "On Lie algebras generated by two differential operators", pp. 187-195 in Manifolds and Lie groups (Notre Dame, IN, 1980), edited by J. Hano et al., Progr. Math. 14, Birkhäuser, Boston, 1981. MR 83e:17013 Zbl 0479.17009

[Kac 1980] V. G. Kac, "Some remarks on nilpotent orbits", J. Algebra 64:1 (1980), 190-213. MR 81i:17005 Zbl 0431.17007

[Kimura 2003] T. Kimura, Introduction to prehomogeneous vector spaces, Translations of Mathematical Monographs 215, American Mathematical Society, Providence, RI, 2003. MR 2003k:11180 Zbl 1035.11060

[Knop 1994] F. Knop, "A Harish-Chandra homomorphism for reductive group actions", Ann. of Math. (2) 140:2 (1994), 253-288. MR 95h:14045 Zbl 0828.22017

[Knop 1998] F. Knop, "Some remarks on multiplicity free spaces", pp. 301-317 in Representation theories and algebraic geometry (Montreal, PQ, 1997), edited by A. Broer and G. Sabidussi, NATO Adv. Sci. Inst. Ser. C Math. Phys. Sci. 514, Kluwer, Dordrecht, 1998. MR 99i:20056 Zbl 0915.20021

[Kostant and Sahi 1991] B. Kostant and S. Sahi, "The Capelli identity, tube domains, and the generalized Laplace transform", Adv. Math. 87:1 (1991), 71-92. MR 92h:22033 Zbl 0748.22008

[Leahy 1998] A. S. Leahy, "A classification of multiplicity free representations", J. Lie Theory 8:2 (1998), 367-391. MR 2000g:22024 Zbl 0910.22015

[Levasseur 2009] T. Levasseur, "Radial components, prehomogeneous vector spaces, and rational Cherednik algebras", Int. Math. Res. Not. 2009:3 (2009), 462-511. MR 2010g:22028 Zbl 1167. 22006

[Levasseur and Stafford 1989] T. Levasseur and J. T. Stafford, "Rings of differential operators on classical rings of invariants", Mem. Amer. Math. Soc. 81:412 (1989). MR 90i:17018 Zbl 0691.16019

[Maßß 1971] H. Maßß, Siegel's modular forms and Dirichlet series, Lecture Notes in Mathematics 216, Springer, Berlin, 1971. MR 49 \#8938 Zbl 0224.10028

[McConnell and Robson 1987] J. C. McConnell and J. C. Robson, Noncommutative Noetherian rings, Wiley, Chichester, 1987. MR 89j:16023 Zbl 0644.16008

[Muller et al. 1986] I. Muller, H. Rubenthaler, and G. Schiffmann, "Structure des espaces préhomogènes associés à certaines algèbres de Lie graduées", Math. Ann. 274:1 (1986), 95-123. MR 88e: $17025 \mathrm{Zbl} 0568.17007$

[Nomura 1989] T. Nomura, "Algebraically independent generators of invariant differential operators on a symmetric cone", J. Reine Angew. Math. 400 (1989), 122-133. MR 91h:22021 Zbl 0667.43007

[Raïs 1972] M. Raïs, "Distributions homogènes sur des espaces de matrices", Mém. Soc. Math. France 30 (1972), 3-109. MR 58 \#22412 Zbl 0245.46045

[Rallis and Schiffmann 1980] S. Rallis and G. Schiffmann, "Weil representation, I: Intertwining distributions and discrete spectrum", Mem. Amer. Math. Soc. 25:231 (1980). MR 81j:22007 Zbl 0442.22006 
[Rubenthaler 2013] H. Rubenthaler, "Multiplicity free spaces with a one-dimensional quotient", $J$. Lie Theory 23:2 (2013), 433-458. MR 3113517 Zbl 1273.14095

[Rubenthaler and Schiffmann 1987] H. Rubenthaler and G. Schiffmann, "Opérateurs différentiels de Shimura et espaces préhomogènes”, Invent. Math. 90:2 (1987), 409-442. MR 89e:11030 Zbl 0638.10024

[Rubenthaler and Schiffmann 1990] H. Rubenthaler and G. Schiffmann, "SL2-triplet associé à un polynôme homogène”, J. Reine Angew. Math. 408 (1990), 136-158. MR 91k:22033 Zbl 0694.17004

[Sato and Kimura 1977] M. Sato and T. Kimura, "A classification of irreducible prehomogeneous vector spaces and their relative invariants", Nagoya Math. J. 65 (1977), 1-155. MR 55 \#3341 Zbl 0321.14030

[Schwarz 2002] G. W. Schwarz, "Finite-dimensional representations of invariant differential operators", J. Algebra 258:1 (2002), 160-204. MR 2004c:16042a Zbl 1020.22005

[Smith 1990] S. P. Smith, "A class of algebras similar to the enveloping algebra of sl(2)", Trans. Amer. Math. Soc. 322:1 (1990), 285-314. MR 91b:17013 Zbl 0732.16019

[Terras 1988] A. Terras, Harmonic analysis on symmetric spaces and applications, II, Springer, Berlin, 1988. MR 89k:22017 Zbl 0668.10033

[Vinberg and Kimelfeld 1978] È. B. Vinberg and B. N. Kimelfeld, "Odnorodnye oblasti na flagovykh mnogoobraziyakh i sfericheskie podgruppy poluprostykh grupp Li”, Funktsional. Anal. i Prilozhen. 12:3 (1978), 12-19. Translated as "Homogeneous domains on flag manifolds and spherical subgroups of semisimple Lie groups”, Funct. Anal. Appl. 12 (1979), 168-174. MR 82e:32042 Zbl 0439.53055

[Wallach 1992] N. R. Wallach, "Polynomial differential operators associated with Hermitian symmetric spaces", pp. 76-94 in Representation theory of Lie groups and Lie algebras (Fuji-Kawaguchiko, 1990), edited by T. Kawazoe et al., World Scientific, River Edge, NJ, 1992. MR 94a:22031 Zbl 1226.22018

[Yan 2000] Z. Yan, "Invariant differential operators and holomorphic function spaces", J. Lie Theory 10:1 (2000), 1-31. MR 2001e:22011 Zbl 0948.43004

Received March 26, 2013. Revised January 8, 2014.

\section{HUBERT RUBENTHALER}

Institut de Recherche Mathématique AvancÉE

UNIVERSITÉ DE STRASBOURG ET CNRS

7 RUE RENÉ DESCARTES

67084 Strasbourg CEDEX

FRANCE

rubenth@math.unistra.fr 


\title{
PACIFIC JOURNAL OF MATHEMATICS
}

\author{
msp.org/pjm
}

Founded in 1951 by E. F. Beckenbach (1906-1982) and F. Wolf (1904-1989)

\section{EDITORS}

Don Blasius (Managing Editor)

Department of Mathematics

University of California

Los Angeles, CA 90095-1555

blasius@math.ucla.edu

\author{
Paul Balmer \\ Department of Mathematics \\ University of California \\ Los Angeles, CA 90095-1555 \\ balmer@math.ucla.edu \\ Robert Finn \\ Department of Mathematics \\ Stanford University \\ Stanford, CA 94305-2125 \\ finn@math.stanford.edu \\ Sorin Popa \\ Department of Mathematics \\ University of California \\ Los Angeles, CA 90095-1555 \\ popa@math.ucla.edu
}

\author{
Vyjayanthi Chari \\ Department of Mathematics \\ University of California \\ Riverside, CA 92521-0135 \\ chari@math.ucr.edu \\ Kefeng Liu \\ Department of Mathematics \\ University of California \\ Los Angeles, CA 90095-1555 \\ liu@math.ucla.edu \\ Jie Qing \\ Department of Mathematics \\ University of California \\ Santa Cruz, CA 95064 \\ qing@ cats.ucsc.edu
}

\section{PRODUCTION}

Silvio Levy, Scientific Editor, production@msp.org

\section{SUPPORTING INSTITUTIONS}

ACADEMIA SINICA, TAIPEI

CALIFORNIA INST. OF TECHNOLOGY

INST. DE MATEMÁTICA PURA E APLICADA

KEIO UNIVERSITY

MATH. SCIENCES RESEARCH INSTITUTE

NEW MEXICO STATE UNIV.

OREGON STATE UNIV.

\author{
STANFORD UNIVERSITY \\ UNIV. OF BRITISH COLUMBIA \\ UNIV. OF CALIFORNIA, BERKELEY \\ UNIV. OF CALIFORNIA, DAVIS \\ UNIV. OF CALIFORNIA, LOS ANGELES \\ UNIV. OF CALIFORNIA, RIVERSIDE \\ UNIV. OF CALIFORNIA, SAN DIEGO \\ UNIV. OF CALIF., SANTA BARBARA
}

\author{
Daryl Cooper \\ Department of Mathematics \\ University of California \\ Santa Barbara, CA 93106-3080 \\ cooper@math.ucsb.edu \\ Jiang-Hua Lu \\ Department of Mathematics \\ The University of Hong Kong \\ Pokfulam Rd., Hong Kong \\ jhlu@maths.hku.hk \\ Paul Yang \\ Department of Mathematics \\ Princeton University \\ Princeton NJ 08544-1000 \\ yang@math.princeton.edu
}

These supporting institutions contribute to the cost of publication of this Journal, but they are not owners or publishers and have no responsibility for its contents or policies.

See inside back cover or msp.org/pjm for submission instructions.

The subscription price for 2014 is US $\$ 410 /$ year for the electronic version, and \$535/year for print and electronic.

Subscriptions, requests for back issues and changes of subscribers address should be sent to Pacific Journal of Mathematics, P.O. Box 4163, Berkeley, CA 94704-0163, U.S.A. The Pacific Journal of Mathematics is indexed by Mathematical Reviews, Zentralblatt MATH, PASCAL CNRS Index, Referativnyi Zhurnal, Current Mathematical Publications and Web of Knowledge (Science Citation Index).

The Pacific Journal of Mathematics (ISSN 0030-8730) at the University of California, c/o Department of Mathematics, 798 Evans Hall \#3840, Berkeley, CA 94720-3840, is published twelve times a year. Periodical rate postage paid at Berkeley, CA 94704, and additional mailing offices. POSTMASTER: send address changes to Pacific Journal of Mathematics, P.O. Box 4163, Berkeley, CA 94704-0163.

PJM peer review and production are managed by EditFLOW ${ }^{\circledR}$ from Mathematical Sciences Publishers.

\section{PUBLISHED BY}

\section{mathematical sciences publishers \\ nonprofit scientific publishing}

http://msp.org/

(C) 2014 Mathematical Sciences Publishers 


\section{PACIFIC JOURNAL OF MATHEMATICS}

Volume $270 \quad$ No. $2 \quad$ August 2014

Disjointification inequalities in symmetric quasi-Banach spaces and 257 their applications

SERgey Astashinin, Fedor A. SukocheV and DMitriy

ZANIN

Hamiltonian evolutions of twisted polygons in parabolic manifolds:

287

The Lagrangian Grassmannian

GLORIA MARÍ BEFFA

On Schwarz-Christoffel mappings

MARTIN CHUAQUi and CHRISTIAN POMMERENKE

Vanishing viscosity in the plane for nondecaying velocity and vorticity, II

ELAINE COZZI

Affine quantum Schur algebras and affine Hecke algebras

QIANG FU

On the classification of Killing submersions and their isometries

JosÉ M. MANZANO

Locally Lipschitz contractibility of Alexandrov spaces and its applications

Ayato Mitsuishi and TAKaO Yamaguchi

Sequences of open Riemannian manifolds with boundary

RAQUEL PERALES and CHRISTINA SORMANI

Invariant differential operators on a class of multiplicity-free spaces 